\title{
Energetic and Broad Band Spectral Distribution of Emission from Astronomical Jets
}

\author{
Asaf Pe'er
}

Received: date / Accepted: date

\begin{abstract}
Emission from astronomical jets extend over the entire spectral band: from radio to the $\mathrm{TeV} \gamma$-rays. This implies that various radiative processes are taking place in different regions along jets. Understanding the origin of the emission is crucial in understanding the physical conditions inside jets, as well as basic physical questions such as jet launching mechanism, particle acceleration and jet composition. In this chapter I discuss various radiative mechanisms, focusing on jets in active galactic nuclei (AGN) and X-ray binaries (XRB) environment. I discuss various models in use in interpreting the data, and the insights they provide.
\end{abstract}

Keywords galaxies: active $\cdot$ gamma-ray bursts $\cdot$ jets $\cdot$ microquasars $\cdot$ radiation mechanism: non-thermal

\section{Introduction}

Jets and outflows are very ubiquitous in astrophysics. They are observed in both galactic objects such as X-ray emitting binaries (XRBs) [For reviews, see, e.g., Fender (2006, 2010); Gallo (2010); Markoff (2010); Maccarone (2012)), as well as extra-galactic sources, such as active galactic nuclei (AGNs) Begelman et al. (1984): Urrv and Padovani (1995); Harris and Krawczynski (2006); Marscher (2009); Ghisellini (2012)], and on a much smaller scale, gamma-ray bursts (GRBs) [e.g., Levinson and Eichler (1993); Piran (2004); Mészáros (2006)]. Recently, the existence of jet was inferred in a tidal disruption event (TDE) of a stray star passing near a massive black hole [Burrows et al. (2011); Levan et al. (2011)]. Emission from jets in both galactic and extra-galactic sources is observed over the entire spectral range: from radio to the highest $\gamma$-rays, at $\mathrm{TeV}$ energies. In addition to the spectral information, in galactic sources (X-ray binaries) as well as in jets from GRBs and TDEs, a wealth of temporal information exists. Similarly, in the high energy ( $\mathrm{GeV}-\mathrm{TeV}$ ) emission from AGNs, flaring activities on time scales

A. Pe'er

Physics Department, University College Cork, Cork, Ireland

Tel.: +353-21-4902594

E-mail: a.peer@ucc.ie 
shorter than $\sim 1$ h has been observed Kniffen et al. (1993); Buckley et al. (1996); Aharonian et al. (2007); Albert et al. (2007a); Aleksić et al. (2011)]. Moreover, in nearby jets from AGNs, such as Cen A, spatial information about emission from different region along the jet exist Hardcastle et al. (2009) and references therein].

While a wealth of data has existed for several decades now, a detailed theoretical understanding of emission from jets is still lacking. One potential explanation is that most work to date has focused on emission from the accreting (inflow) material, and only in the past decade or so have more advanced models of emission from the outflow (jets) emerged. One notable exception is the emission from GRB jets: as our understanding of these objects relies nearly entirely on studying emission from their jets, the theory of emission from GRB jets is likely the most advanced one to date. A second reason is the enormous complexity of these systems. As will be discussed here, although the nature of the radiative processes is well understood, as the physical conditions inside and in the vicinity of the jets are poorly constrained, the data can be interpreted in more than one way. As a result, a plethora of models exist, and a conclusive picture is still lacking.

Although different objects share the common property of having jets, there is a huge difference in scales of the observed objects. While XRBs and GRBs are stellar-size objects, with a typical mass of the central $\mathrm{BH}$ of $\sim$ few $M_{\odot}$, the black holes in the center of AGNs have masses of $10^{6}-10^{9} M_{\odot}$. This difference in scaling results in very different scales of the resulting jets. In galactic XRBs the inferred size of the observed jets is typically 100's of AU's $\left(\gtrsim 10^{14}-10^{15} \mathrm{~cm}\right)$ Miller-Jones et al. (2012)] while radio 'blobs' are seen on much larger, sub-pc scales $\left(\sim 10^{17} \mathrm{~cm}\right)$. In GRBs, the jet does not deposit most of its energy in the environment before reaching $\sim 10^{18} \mathrm{~cm}$ Meszaros and Rees (1997); Wijers et al. (1997)], although analysis shows that emission exists from the photosphere at $\sim 10^{12} \mathrm{~cm}$ Axelsson et al. (2012)]. Sizes of AGN jets extend to much larger scales, with giant radio lobes extending to hundreds of $\mathrm{kpc}, \sim 10^{23} \mathrm{~cm}$ Alvarez et al. (2000) and references therein].

This difference in scaling implies that the physical conditions, and hence the leading radiative mechanisms inside the jets, vary with distance. Nonetheless, the basic emission processes are the same in all sources. The leading radiative processes include synchrotron emission, synchrotron self-Compton and Compton scattering of photons external to the jet - either photons originating from the accretion disk, companion star (in XRBs) or from the cosmic microwave (or infra-red) background (CMB). If hadrons (mainly protons) are accelerated to high energies in jets, they can also make a significant contribution to the emission, particularly at high energies ( $\mathrm{X}$ and $\gamma$-ray bands). This contribution is both by direct emission( e.g., synchrotron), and indirectly, by interacting with photons and protons to produce secondaries (pions, Kaons and electron-positron pairs) which contribute to the emitted spectra. In addition, emission from the photosphere, defined here as emission that originates from regions in space in which the optical depth of photons to reach the observer is larger than unity, may play an important role.

In addition to the spectral analysis, two very important sources of information exist. The first is temporal variability which provides strong constraints on the physical conditions inside the jets, and hence on the emission processes. This played a crucial role in the development of the leading theory of emission from GRB jets (the "fireball" model). Studying the correlated variability seen in the emission at different wavelengths (radio /IR/optic and $\mathrm{X} / \gamma$-rays) in XRBs is likely the key to 
understanding of the emission from these objects [See Uttley et al. (2011), and the chapters by Gallo and Casella in this book]. The second source of information is spatial analysis, which is particularly useful when studying the largest-scale jets in AGNs. The "hot spots" frequently seen imply that the physical conditions and the radiative processes vary along the jet. Thus, a full physical picture must take into account first the dynamics of the outflow and second the radiation. Clearly, both parts are connected, and, in addition, give information about the jet launching process and the properties of the inner accretion disk.

Another important factor that needs to be considered in analyzing the emission is geometry. There are several aspects to this issue. First, as astronomical jets are mildly relativistic in XRBs $(\Gamma \gtrsim f e w)$, often relativistic in $\operatorname{AGNs}(\Gamma \gtrsim 10)$ and highly relativistic in GRBs $(\Gamma \gtrsim 100-1000)$, relativistic Doppler effect is important when analyzing the spectrum, as the jets will rarely point directly towards us. Emission from relativistically expanding blobs can lead to an apparent motion faster than the speed of light (a phenomenon known as "superluminal motion" Rees (1966); Mirabel and Rodríguez (1994)]). Second, jets, by definition, have spatial structure (often referred to as "structured jets"): a velocity profile exists, namely $v=v(r, \theta, \phi)$, where the angles $\theta, \phi$ are measured relative to the jet axis. Thus, a velocity gradient in the transverse direction (perpendicular to the jet propagation direction) exists, with an obvious effect on the scattering between electrons and photons, and hence on the observed spectra.

Finally, the velocity structure in the radial direction can lead to confusing definition of jets. One possibility is that the outflow is continuous (generating a smooth velocity gradient in the radial direction) in which case it will be seen as a continuous jet. Alternatively, the outflow may be fragmented: in this case, the outflow will be observed as 'blobs' that propagate outward, while expanding (possibly, but not necessarily, adiabatically). Of course, the observed emission from these blobs imply that the conditions inside the blobs are different than those outside. Thus, when studying emission from these blobs one needs to consider the conditions both inside the blobs and in the surrounding material. In this chapter, we will treat both emission from continuous jets as well as from the blobs.

Thus, a full description of the emission requires understanding of (1) the dynamics, (2) the geometry and (3) the various radiative processes. Clearly, I cannot possibly cover the entire physics of jet emission in one chapter. I will thus focus on key radiative processes. I will show how the basic, well-known radiative processes can lead to the wealth of spectra observed. I will also try to point to basic, unsolved questions which naturally arise when analyzing the emission. The discussion will be focused on the jet emission from XRB and AGN environments, which show several similar key properties, although having different scales. Clearly, many of the results are relevant to jets in GRBs and TDEs as well.

\section{Basic radiative processes: synchrotron emission}

Variable radio emission in AGNs and XRBs is conventionally interpreted as synchrotron radiation from a non-thermal distribution of relativistic electrons. Indeed, synchrotron emission, being perhaps the most straightforward emission mechanism for explaining non-thermal radiation has been extensively studied since the 1960's 
Ginzburg and Syrovatskii (1965); Blumenthal and Gould (1970)]. Two basic ingredients are needed: energetic particles and a strong magnetic field.

Consider a source at redshift $z$ which is moving at velocity $\beta \equiv v / c$ (corresponding Lorentz factor $\Gamma=(1-\beta)^{-1 / 2}$ ) at angle $\theta$ with respect to the observer. The emitted photons are thus seen with a Doppler boost $\mathcal{D}=[\Gamma(1-\beta \cos \theta)]^{-1}$. Synchrotron emission from electrons having random Lorentz factor $\gamma_{e l}$ in a magnetic field $B$ (all in the comoving frame) is observed at a typical energy

$$
\varepsilon_{m}^{o b}=\frac{3}{2} \hbar \frac{q B}{m_{e} c} \gamma_{e l}^{2} \frac{\mathcal{D}}{(1+z)}=1.75 \times 10^{-19} B \gamma_{e l}^{2} \frac{\mathcal{D}}{(1+z)} \mathrm{erg} .
$$

Thus, when studying this emission, the basic physical questions are:

1. What is the origin of the magnetic field ?

2. What is the mechanism that accelerates particles to high energies? Does this mechanism accelerate only electrons? Are protons being accelerated similarly, thereby contributing to the emission? What is the resulting energy distribution of the energetic particles, $n(E) d E$ ?

3. What is the spatial / temporal evolution of the magnetic field and particle distribution in different regions along the jet?

While significant progress has been made in the last few decades, proper understanding of any of these issues remains elusive. These questions are deeply related to the physics of the jet launch mechanism, and jet composition. While there is no direct observational test that addresses any of these phenomena, very considerable theoretical effort supported by state of the art numerical simulations, as well as indirect interpretation of existing data, all suggest that these questions are likely intimately related to each other. We discuss these questions in what follows.

Origin of magnetic field. Although the question of magnetic field generation is a fundamental one, little is known about the exact mechanism at work in these objects. Roughly speaking, there are two main (separated) sources of strong magnetic fields: the first is related to the accretion flow and the jet launching process. Possibly, even if the magnetic fields do not carry a large fraction of the kinetic energy, they may still play a key role in jets collimation. The second, independent source is magnetic field generation in shock waves that exist inside the outflow itself (assuming it is irregular), or when the outflow interacts with its surroundings - the interstellar medium (ISM) or intergalactic medium (IGM). A third possible source is amplification of ISM or IGM magnetic fields when compressed by the expanding shock waves, but in this case the amplified fields can at most explain the observed emission in the interaction of the outflow with its surroundings they are much too weak to be consistent with the ones required to explain the observed properties in the inner jet regions. For a recent review on magnetic fields in astrophysical jets, see Pudritz et al. (2012).

The two leading mechanisms believed to operate for jet launching are the Blandford and Znaiek (1977) and Blandford and Payne (1982) mechanisms. In the Blandford and Znajek (1977) mechanism, the source of energy is the rotational energy extracted from a rotating black hole, embedded in a strong magnetic field. The field itself must be anchored into the accretion flow Livio et al. (1999); Meier (2001)]. In the Blandford and Pavne (1982) mechanism, energy is extracted from the accretion disk by magnetic field lines that leave the disk surface and extend to large distances. This is accompanied by centrifugally-driven outflow of material 
from the inner parts of the disk, that is attached to the field lines [for further explanation see Spruit (2010), as well as the chapter on jet acceleration in this book]. Both mechanisms require a strong magnetic field attached to the disk. At larger distances along the jet, the magnetic field decays as Poynting flux is conserved.

These ideas have been recently tested and validated with state of the art numerical GR-MHD simulations Meier et al. (2001); McKinney and Gammie (2004); McKinney (2005, 2006); Tchekhovskoy et al. (2010, 2011)]. These models imply that the magnetic field originates in the inner parts of the disk. The inner parts of the jets are strongly magnetized (Poynting-flux dominated), and the magnetic energy is gradually dissipated along the jet. The dissipated energy is then used to accelerate the particles along the jet [see, e.g., Vlahakis and Königl (2004); Komissarov et al. (2007)]. Recent observations on parsec-scale in AGNs indicate magnetic field strengths consistent with those expected from theoretical models of magnetically powered jets O'Sullivan and Gabuzda (2009)]. However, the picture is far more complicated, since modeling the broad-band emission (radio - X-rays) on a $\gtrsim$ parsec scale from several AGNs show that the magnetic field must be subdominant, and most of the kinetic energy is carried by protons (particle-dominated jets) Celotti and Fabian (1993); Krawczynski et al. (2002); Kino et al. (2002); Celotti and Ghisellini (2008)]. The mechanism in which magnetic-dominated outflow at the core becomes particle dominated at larger distances is far from being clear.

Independent of the question of jet launching, a second source of strong magnetic fields are shock waves that develop as a result of instabilities within the outflow. These shock waves can result, e.g., from fluctuations in the ejection process itself: if a slower moving plasma shell (or "blob") is followed by a faster moving one, the two shells will eventually collide, producing a pair of forward and reverse shock waves propagating into each of these blobs. These shock waves may generate strong magnetic fields by two-stream instabilities Weibel (1959); Medvedev and Loeb (1999)]. In recent years, advances in particle-in cell (PIC) simulations enabled to study this process by tracing the instability growth modes Silva et al. (2003); Frederiksen et al. (2004); Nishikawa et al. (2005); Spitkovsky (2008a)]. The results of these works have demonstrated that strong magnetic fields are indeed created in collisionless shock waves.

The key question though, is the decay length of the magnetic field: the results discussed above also show that the generated field decays on a very short length scale, of the order of few hundred skin depths [Spitkovsky (2008a)]. As observations imply that the emitting region is many orders of magnitude larger than this scale, there must be a mechanism that maintains a strong magnetic field extending to much larger scales. One suggestion is that the amplification of the magnetic field may be closely related to the acceleration of particles to high energies Keshet et al. (2009)]. Thus, while initially the magnetic field may occupy only a small region close to the shock front, over time, as particles are accelerated to increasingly higher energies, the magnetized region expands. This suggestion is difficult to directly test, due to the numerical complexity of the problem. Another suggestion is that, due to the turbulent nature of the post-shock outflow, the magnetic field is maintained over a long distance behind the shock front Zrake and MacFadyen (2012)]. Thus, while it is clear that magnetic fields can be generated in shock 
waves, the exact scaling (strength and decay length) of these fields is still a matter for debate.

Particle acceleration. It should be emphasized that the existence of cosmic rays, [charged particles that are observed at energies as high as $\gtrsim 10^{20} \mathrm{eV}$; for a recent review, see Kotera and Olinto (2011)], is a direct evidence that particle acceleration to ultra-high energies takes place in astronomical objects. However, there is no direct information on the exact nature of the cosmic ray sources, nor on the nature of the acceleration process itself. Hence the question of lepton (electrons and positrons) acceleration is inferred indirectly, by fitting the observed spectra from various objects. It is most likely that acceleration takes place in several different locations: in the nucleus, in the hot spots and possibly additional locations along the jet axis.

The most widely discussed mechanism for acceleration of particles is the Fermi mechanism Fermi (1949, 1954)], which requires the particles to cross back and forth a shock wave. A basic explanation of this mechanism can be found in the textbook by Longair. For reviews see Bell (1978); Blandford and Ostriker (1978); Blandford and Eichler (1987); Jones and Ellison (1991). In this process, the accelerated particle crosses the shock multiple times, and in each crossing its energy increases by a (nearly) constant fraction, $\Delta E / E \sim 1$. This results in a power law distribution of the accelerated particles, $N(E) \propto E^{-S}$ with power law in$\operatorname{dex} S \approx 2.0-2.4$ Kirk et al. (1998, 2000); Ellison et al. (1990); Achterberg et al. (2001); Ellison and Double (2004)]. Recent developments in particle-in-cell (PIC) simulations have allowed to model this process from first principles, and study it in more detail Silva et al. (2003); Nishikawa et al. (2003); Spitkovsky (2008b); Sironi and Spitkovsky (2009); Haugbølle (2011)]. However, due to the numerical complexity of the problem, these simulations can only cover a tiny fraction $\left(\sim 10^{-8}\right)$ of the actual emitting region in which energetic particles exist. Thus, these simulations can only serve as guidelines, and the problem is still far from being fully resolved. Regardless of the exact details, it is clear that particle acceleration via the Fermi mechanism requires the existence of shock waves, and is thus directly related to the internal dynamics of the gas inside the jet, and possibly to the generation of magnetic fields, as mentioned above.

An alternative model for particle acceleration is magnetic reconnection. The basic idea is that when magnetic field lines change their topology and form a reconnection layer, magnetic energy is released. Part of the generated energy may be used to accelerate particles to high energies Romanova and Lovelace (1992); Lyutikov (2003); Lyubarsky and Liverts (2008); Lazarian et al. (2011); McKinney and Uzdensky (2012)]. This idea is very appealing if jets are highly magnetized (at least close to the core), as is suggested by the leading theories of jet launching. In fact, it is not clear that the conditions that enable particle acceleration to high energies in shock waves exist at all in highly magnetized outflows Sironi and Spitkovsky (2009, 2011)], in which case the Fermi mechanism may not be at work. However, theoretical understanding of this process, and its details (e.g., what fraction of the reconnected energy is being used in accelerating particles, or the energy distribution of the accelerated particles) is still very limited.

Although the power law distribution of particles resulting from Fermi-type, or perhaps magnetic-reconnection acceleration is the most widely discussed, we point out that alternative models exist. One such model involves particle acceleration by a strong electromagnetic potential, which can exceed $10^{20} \mathrm{eV}$ close to the jet 
core Lovelace (1976); Blandford (1976); Neronov et al. (2009)]. The accelerated particles may produce a high energy cascade of electron-positron pairs. Additional model involves stochastic acceleration of particles due to resonant interactions with plasma waves in the black hole magnetosphere [Dermer et al. (1996)].

Several authors have also considered the possibility that particles in fact have a relativistic quasi-Maxwellian distribution Jones and Hardee (1979); Cioffi and Jones (1980); Wardziński and Zdziarski (2000); Pe'er and Casella (2009)]. Such a distribution, with the required temperature $\left(\sim 10^{11}-10^{12} \mathrm{~K}\right)$ may be generated if particles are roughly thermalized behind a relativistic strong shock wave [e.g., Blandford and McKee (1977)]. Interestingly, this model is consistent with several key observations, as will be discussed below.

Spatial and temporal distributions. The uncertainty that exists in both the origin of the magnetic field as well as the nature of the particle acceleration process is directly translated to an uncertainty in the spatial and temporal distributions of these two quantities, and hence on the emission pattern. If the magnetic field originates in the disk, then as the jet expands the magnetic field must decay. For example, if the cross sectional radius of the jet is $r=r(z)$ where $z$ is the direction along the jet axis, then Poynting flux conservation implies $B \propto r^{-1}$. If, on the other hand, the dominant process for magnetic field generation is two stream instability in shock waves, the magnetic field then traces the shock wave location. Thus, strong magnetic fields are expected only above a certain radius, where plasma shells collide. This is very likely the case in the spatially resolved "radio blobs" seen in XRBs, as well as in "knots" observed along AGN jets.

The magnetic field strength may also be different in the two possibilities discussed. Lacking a complete theory, it is commonly assumed that the generated magnetic field carries a constant fraction, $\epsilon_{B}$ of the kinetic energy dissipated by the shock wave, $B^{2} / 8 \pi=\epsilon_{B} U$. Here, $U$ is the (post-shock) energy density of the plasma. Estimated values for $\epsilon_{B}$ based on fitting the data vary from equipartition $\left(\epsilon_{B}=1 / 3\right.$; Miller-Jones et al. (2005); Cerruti et al. (2013)]) to $\epsilon_{B} \sim 10^{-2}$, possibly even lower Celotti and Ghisellini (2008); Santana et al. (2013)].

The spatial and temporal distribution of the energetic particles is determined by several factors. Once accelerated to high energies, the radiating particles lose their energy both adiabatically as the jet expands, and radiatively, as they radiate their energy. Thus, in order to understand their spatial distribution, one needs to know (1) the initial distribution of the energetic particles accelerated by the acceleration process (determined by the unknown nature of this process). (2) The dynamics of the plasma; and (3) the physical conditions inside the plasma, that govern the energy loss rate.

\subsection{Spectral shape: basic considerations and Maxwellian distribution of electrons}

The discussion above points towards high uncertainty in our knowledge of the initial energy distribution of particles produced by the acceleration process. It is commonly believed that the acceleration process produces a power law distribution $n(E) d E \propto E^{-S}$, with $S \approx 2.0-2.4$. This is based on (1) theoretical expectations from Fermi acceleration, and (2) interpretation of broad band synchrotron emission. However, a few words of caution are necessary here. First, as discussed above, it is not clear that the Fermi process is necessarily the acceleration mechanism at 
work in these objects. Second, as was recently shown Pe'er and Casella (2009)] and will be discussed below, the observed data can be interpreted in a way that does not require a power law distribution of the radiating particles. Thus, evidence for the existence of a power law distribution is inconclusive.

Even if the acceleration process is indeed Fermi-type in shock waves, then the resulting power law distribution is expected to be limited to a certain region in energy space. As particles cross the shock front, they thermalize. Strong shock waves propagating at Lorentz factor $\Gamma$ into a cold material of density $n$ compress the material so that its density in the downstream region is $4 \Gamma n$. The material is being heated: the energy density in the downstream region is $4 \Gamma^{2} n m_{p} c^{2}$. Thus, the average energy per particle in the downstream region is $\approx \Gamma m_{p} c^{2}$ Blandford and McKee (1976) $)$. If a fraction $\epsilon_{e} \leq 1$ of this energy is carried by the energetic electrons, then (neglecting a possible contribution from pairs) the expected Lorentz factor of the electrons is $\gamma_{e l} \approx \Gamma \epsilon_{e} m_{p} / m_{e}$. Note that this is the Lorentz factor associated with the random motion of the electrons as they cross the shock front, and should not be confused with the Lorentz factor associated with the bulk motion of the flow, which is of the order of $\Gamma$. Thus, even in mildlyrelativistic outflows $(\Gamma \gtrsim 1)$, the electrons in the downstream region may still have (random) Lorentz factor of few hundreds, provided that $\epsilon_{e}$ is close to equipartition.

One can thus conclude that regardless of the question of whether electrons are accelerated to a power law distribution, they are still expected to be heated to high energies (high Lorentz factors) when shock waves exist. Hence, if no further acceleration process are present, the electrons will have a Maxwellian distribution with typical Lorentz factor $\gamma_{e l} \lesssim 10^{3}$ (assuming $\epsilon_{e}$ close to equipartition). In the vicinity of a magnetic field $B$, which could naturally be generated by the same shock wave, electrons at the peak of the Maxwellian distribution will emit at a characteristic energy given by Equation[1. For typical value $\gamma_{e l} \sim 10^{3}$ and $B \sim 1 \mathrm{G}$, Equation 1 implies a characteristic observed frequency in the optical band.

This result implies that in order to explain the observed flat radio spectra seen in many objects, there is no need to invoke a power law distribution of the accelerated particles. It is enough to consider a power law decay of the magnetic field along the jet, $B(r) \propto r^{-\alpha}$ to obtain a power law decay of the peak synchrotron frequency below the optical band, in accordance to Equation 1 . A power law spectrum would be observed if the emission is not spatially resolved, but is integrated over some distance along the jet along which the magnetic field decays. This is a typical scenario for the inner parts of AGN jets, as well as for jets in XRBs.

\subsection{Power law distribution of the accelerated particles}

It is possible to envision a different model, in which the energy distribution of the accelerated electrons is a power law. In fact, historically this model was the first to be suggested in explaining the observed spectra van der Laan (1966); Blandford and Konigl (1979)], and is still the most widely-discussed one.

An uncertainty lies in the fraction of particles that are being accelerated: as the electrons cross the shock wave, they have a thermal distribution with typical Lorentz factor $\gamma_{e l}$. As some fraction continues to cross the shock front multiple times, this fraction obtain a power law distribution. What fraction of particles are accelerated to a power law distribution above the Maxwellian is unclear. Recent 
PIC simulations suggest that only a small fraction of the population, $\epsilon_{p l} \approx 1 \%-$ $10 \%$ form a power law tail at higher energies [Spitkovsky (2008b)]. However, as discussed above, these conclusions are far from being certain, and it is possible that the fraction is much higher, perhaps even closer to $100 \%$.

A theoretical limit on the maximum energy is obtained by the requirement that the acceleration time must be shorter than the minimum energy loss time (e.g., due to synchrotron radiation or Compton scattering) and the time in which the accelerated particle is confined to the accelerated region. In a plasma which moves relativistically with Lorentz factor $\Gamma$, the acceleration time in Fermi-type acceleration is [e.g., Norman et al. (1995)]

$$
t_{a c c}=\frac{\eta E^{\mathrm{ob}}}{\Gamma Z q B c}
$$

Here, $Z q$ is the charge of the particle (the same equation holds for electrons, protons as well as heavy nuclei with $Z$ protons), and $E^{\mathrm{ob}}$ is the energy of the energetic particle in the observer's frame. The exact value of the dimensionless factor $\eta \geq 1$ depends on the uncertain details of the acceleration process: for example, in nonrelativistic diffusive shock acceleration, this factor corresponds to $\eta=(20 / 3) \beta^{-2}$ in the Bohm limit for parallel shocks [e.g., Blandford and Eichler (1987)].

The second requirement constraints the size of the acceleration region. For typical values of parameters that govern the emission in XRBs and AGNs, it is not very restrictive. On the other hand, the requirement that the acceleration time is shorter than the radiative cooling time puts a stronger constraint on the maximum energy of the accelerated particles. The radiative cooling time of energetic electrons due to synchrotron emission and Compton scattering is

$$
t_{\mathrm{cool}}=\frac{E}{P}=\frac{\gamma_{e l} m_{e} c^{2}}{(4 / 3) c \sigma_{T} \gamma_{e l}^{2} u_{B}(1+Y)}=\frac{6 \pi m_{e} c}{\sigma_{T} B^{2} \gamma_{e l}(1+Y)},
$$

where $u_{B} \equiv B^{2} / 8 \pi$ is the energy density in the magnetic field, $\sigma_{T}$ is Thomson's cross section and $Y$ is Compton parameter. Comparing the radiative cooling time in Equation 3 to the acceleration time in Equation 2 gives a theoretical upper limit on the energy of the accelerated electrons,

$$
\gamma_{\max }=\left(\frac{6 \pi q}{\eta B \sigma_{T}(1+Y)}\right) .
$$

Using the derived value of $\gamma_{\max }$ from Equation 4 in Equation 1 gives a very interesting result: the characteristic energy of photons emitted by these electrons,

$$
\varepsilon_{\max }^{\mathrm{ob}}=240 \frac{\mathcal{D}}{\eta(1+Y)(1+z)} \mathrm{MeV}
$$

is independent on the strength of the magnetic field. This result implies that regardless of the value of the magnetic field, if indeed particles are accelerated by a Fermi-type mechanism in shock waves, synchrotron emission is expected to be observed at all energies up to the $\gamma$-ray band. Thus, it is possible, at least from a theoretical perspective, that synchrotron photons have a significant contribution to the emission at $\mathrm{X}$ and $\gamma$-ray energies. 
2.3 Broad band, spatially resolved synchrotron spectrum

The discussion above implies that even if synchrotron emission is the only source of radiation, and even if the magnetic field is constant, the complex distribution of the energetic particles leads to a complex observed spectrum. In addition to the two frequencies discussed, $\nu_{m}=\varepsilon_{m} / h$ (see Equation 11) and $\nu_{\max }=\varepsilon_{\max } / h$ (the later exists only if the acceleration process produces a power law), there are additional two inherent characteristic frequencies. The first is the synchrotron self absorption frequency, $\nu_{S S A}$, below which synchrotron photons are absorbed. This frequency can be either above or below $\nu_{m}$. The exact value of $\nu_{S S A}$ depends on the magnetic field strength and the distribution of the radiating particles [for discussion see Rybicki and Lightman (1979)]. For typical parameters, $\nu_{S S A}<\nu_{m}$. However, given the uncertainty that exists in the acceleration process and the strength of the magnetic fields, it is possible to envision scenarios in which $\nu_{m}<\nu_{S S A}$.

The fourth frequency is the cooling frequency, $\nu_{c}$. This is the characteristic emission frequency from particles whose radiative cooling time (given by Equation 3) is equal to the characteristic plasma expansion time, $t_{d y n} \simeq r / \Gamma c$, where $r$ is the radius of the expanding plasma. Since the cooling time is inversely proportional to the particle's Lorentz factor, $t_{\text {cool }} \propto \gamma^{-1}$ (see Equation 3), energetic particles cool faster than low energy ones. Above a certain Lorentz factor, denoted by $\gamma_{c}$, particles cool faster than the dynamical time. Thus, if particles are accelerated only once, $\gamma_{c}=\gamma_{\max }$. However, if the acceleration continuously produces a power law distribution of energetic particles, $n(\gamma) d \gamma \propto \gamma^{-S}$, then $\gamma_{c}$ marks a transition in the steady state distribution. By solving the continuity equation, it is easy to show [see, e.g., Longair] that for $\gamma \gg \max \left(\gamma_{c}, \gamma_{m}\right)$, the steady particle distribution is $n(\gamma) d \gamma \propto \gamma^{-(S+1)}$. If $\gamma_{c}<\gamma_{e l}$, then in the region $\gamma_{c} \ll \gamma \ll \gamma_{e l}$ the steady distribution is $n(\gamma) d \gamma \propto \gamma^{-2}$. This break in the particle distribution is directly translated to a break in the emitted spectrum. As the synchrotron spectrum from a power law distribution of particles with power law index $S$ is $F_{\nu} \propto \nu^{-(S-1) / 2}$, at frequencies above $\nu_{c}$, this power law changes to $F_{\nu} \propto \nu^{-S / 2}$.

Thus, in Fermi-type acceleration, four breaks in the spectrum are expected (see Table 1). Even if the acceleration mechanism produces only a Maxwellian distribution of hot particles, at least two break frequencies $\left(\nu_{S S A}\right.$ and $\left.\nu_{m}\right)$ are unavoidable.

Table 1 Key break frequencies

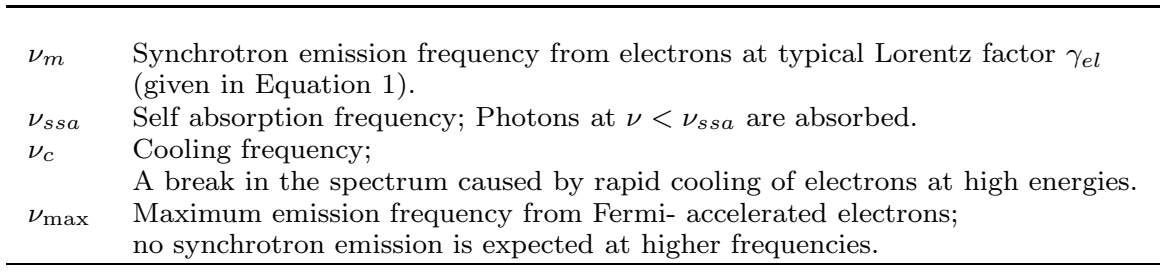

In the scenario where $\nu_{S S A}<\nu_{m}<\nu_{c}$ the peak of the spectrum occurs at $\nu=\nu_{m}$. Denoting by $F_{\nu, \max }$ the observed peak flux, the broad band synchrotron 

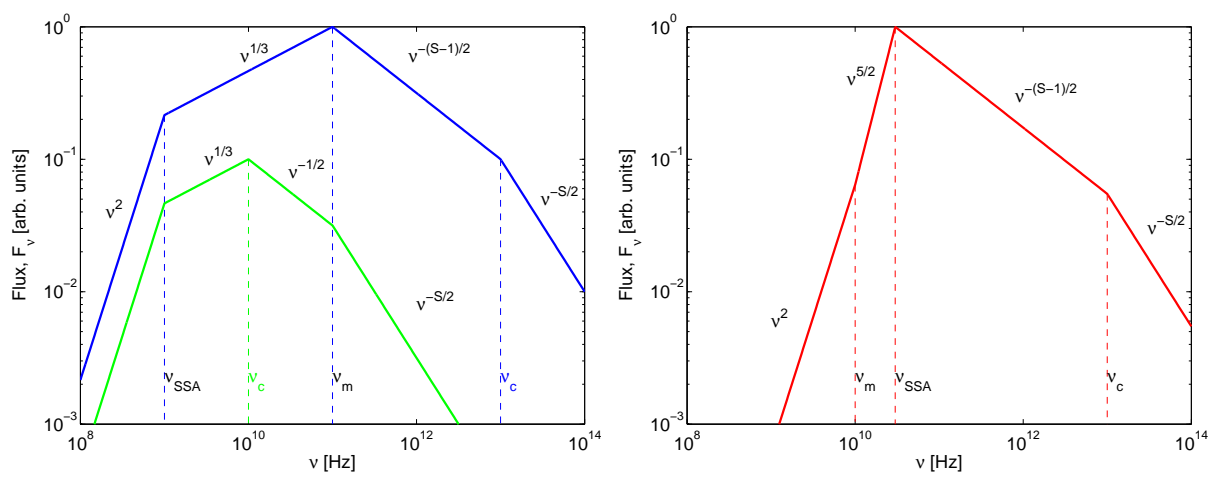

Fig. 1 Broad band synchrotron spectra from a power law distribution of energetic particles in a steady magnetic field, as expected from a small jet slab. The flux and the chosen values of the break frequencies are arbitrary, and depend on the exact values of the magnetic field, particles energies and number of radiating particles. Left: the spectra expected when $\nu_{S S A}<\nu_{m}<\nu_{c}$ (blue) peaks at $\nu_{m}$. In the case $\nu_{S S A}<\nu_{c}<\nu_{m}$ (green), the spectrum peaks at $\nu_{c}$. These spectra were considered in the model of Pe'er and Casella (2009). Right: the broad band spectra in the scenario $\nu_{m}<\nu_{S S A}<\nu_{c}$ peaks at $\nu_{S S A}$. This is the scenario considered in the model of Blandford and Konigl (1979), and is commonly considered in the literature thereafter.

spectrum is [e.g., Meszaros and Rees (1997); Sari et al. (1998)]

$$
F_{\nu}=F_{\nu, \max } \times\left\{\begin{array}{lc}
\left(\nu / \nu_{S S A}\right)^{2}\left(\nu_{S S A} / \nu_{m}\right)^{1 / 3} & \nu<\nu_{S S A} \\
\left(\nu / \nu_{m}\right)^{1 / 3} & \nu_{S S A}<\nu<\nu_{m} \\
\left(\nu / \nu_{m}\right)^{-(S-1) / 2} & \nu_{m}<\nu<\nu_{c} \\
\left(\nu_{c} / \nu_{m}\right)^{-(S-1) / 2}\left(\nu / \nu_{c}\right)^{-S / 2} & \nu_{c}<\nu<\nu_{\max }
\end{array}\right.
$$

If, on the other hand $\nu_{S S A}<\nu_{c}<\nu_{m}$, the peak of the emission is at $\nu_{c}$, and the spectral shape is

$$
F_{\nu}=F_{\nu, \max } \times\left\{\begin{array}{lc}
\left(\nu / \nu_{S S A}\right)^{2}\left(\nu_{S S A} / \nu_{c}\right)^{1 / 3} & \nu<\nu_{S S A} \\
\left(\nu / \nu_{c}\right)^{1 / 3} & \nu_{S S A}<\nu<\nu_{c} \\
\left(\nu / \nu_{c}\right)^{-1 / 2} & \nu_{c}<\nu<\nu_{m} \\
\left(\nu_{m} / \nu_{c}\right)^{-1 / 2}\left(\nu / \nu_{m}\right)^{-S / 2} & \nu_{m}<\nu<\nu_{\max }
\end{array} .\right.
$$

Finally, the model of Blandford and Konig] (1979) can be viewed as a model in which $\nu_{m}<\nu_{s s a} \ll \nu_{c}$. In this case, the peak of the emission is at $\nu_{S S A}$, and the broad band spectrum is

$$
F_{\nu}=F_{\nu, \max } \times\left\{\begin{array}{lc}
\left(\nu / \nu_{m}\right)^{2}\left(\nu_{m} / \nu_{S S A}\right)^{5 / 2} & \nu<\nu_{m} \\
\left(\nu / \nu_{S S A}\right)^{5 / 2} & \nu_{m}<\nu<\nu_{S S A} \\
\left(\nu / \nu_{S S A}\right)^{-(S-1) / 2} & \nu_{S S A}<\nu<\nu_{c} \\
\left(\nu / \nu_{c}\right)^{-S / 2}\left(\nu_{c} / \nu_{S S A}\right)^{-(S-1) / 2} & \nu_{c}<\nu<\nu_{\max }
\end{array}\right.
$$

These spectra are shown in Figure 1. 
2.4 Integrated spectrum: flat radio emission

The broad band spectrum considered above is developed under the assumptions that the radiating particles have a power law distribution and that the magnetic field is steady. In reality, once accelerated, or even during the acceleration the energetic particles propagate along the jet. As the magnetic field strength varies along the jet, the break frequencies $-\nu_{S S A}, \nu_{m}$ and $\nu_{c}$ (but not $\nu_{\max }$ !) are different in different regions along the jet. If the jet is spatially resolved, this implies that different regions along the jet are characterized by different spectra. If the jet is spatially unresolved, as is the case in XRBs and the inner parts of jets in AGNs, then the observed spectrum is obtained by integrating over different emission regions, each characterized by different break frequencies. This integration naturally leads to the observed power law spectra, such as the flat spectra frequently observed at radio frequencies, in both AGNs (the so called "flat spectrum radio quasars", or FSRQ), and more recently in XRBs Hynes et al. (2000); Fender (2001, 2006), and chapters in this book by Fender, Gallo and Casella].

If the origin of the magnetic field is in the disk, it is expected to decay along the jet as a power law with distance. This decay, which results in a corresponding decrease of the break frequencies, is all that is needed to produce a power law spectrum in the radio band, in particular the flat spectrum that is typically observed. This was first noted by Blandford and Konigl (1979). In this model, a conical jet with $B(r) \propto r^{-1}$ and steady outflow velocity resulting in a number density variation along the jet $n(r) \propto r^{-2}$ was analyzed. Only the evolution of the self absorption frequency, $\nu_{S S A}$ along the jet was considered. In the more general framework considered here, this is equivalent to a model in which particles are accelerated to a power law energy distribution with $\nu_{m}\left\langle\nu_{S S A} \ll \nu_{c}\right.$ as is presented in Figure 1 (right). In this scenario, the emission from a jet slab (in which the magnetic field is constant), peaks at $\nu_{S S A}$. It is straightforward to show [see, e.g., Rvbicki and Lightman (1979); Blandford and Konigl (1979)] that these conditions lead to a decay of the self absorption frequency along the jet, $\nu_{S S A} \propto r^{-1}$, while the flux from a slab along the jet axis at the self absorption frequency is constant, $\left.d F_{\nu}\right|_{\nu_{S S A}} \propto \nu^{0}$. Thus, when integrated over an unresolved distance along the jet a flat radio spectrum is obtained. This is demonstrated in Figure 2, taken from Markoff (2010).

This basic idea was extended by several authors in various aspects. For example, Marscher (1980) considered different viewing angles, while Revnolds (1982) considered different dynamical models for the outflow. In another work, Hiellming and Johnston (1988) considered a more refined jet geometry, as well as adiabatic (though, not radiative) energy losses. Several works (Falcke and Biermann (1995); Levinson and Blandford (1996); Falcke and Biermann (1999); Heinz and Sunvaev (2003); Bosch-Ramon et al. (2006)) have connected the jet properties to the disk properties, and refined the inner jet dynamics. This dynamics was used by several authors Markoff et al. (2001, 2003, 2005); Yuan et al. (2005); Yuan and Cui (2005); Maitra et al. (2009)] to model the broad band spectra of XTE J1118+480 and GX 339-4. These works included a self consistent modeling of the emission from the radio all the way to the X-ray band. In modeling the X-ray emission, the power law assumption was used to fit both the synchrotron emission and the synchrotron self Compton (SSC) emission Markoff et al. (2001); Gallo et al. (2007); Migliari et al. (2007); Maitra et al. (2009); see further discussion below]. 


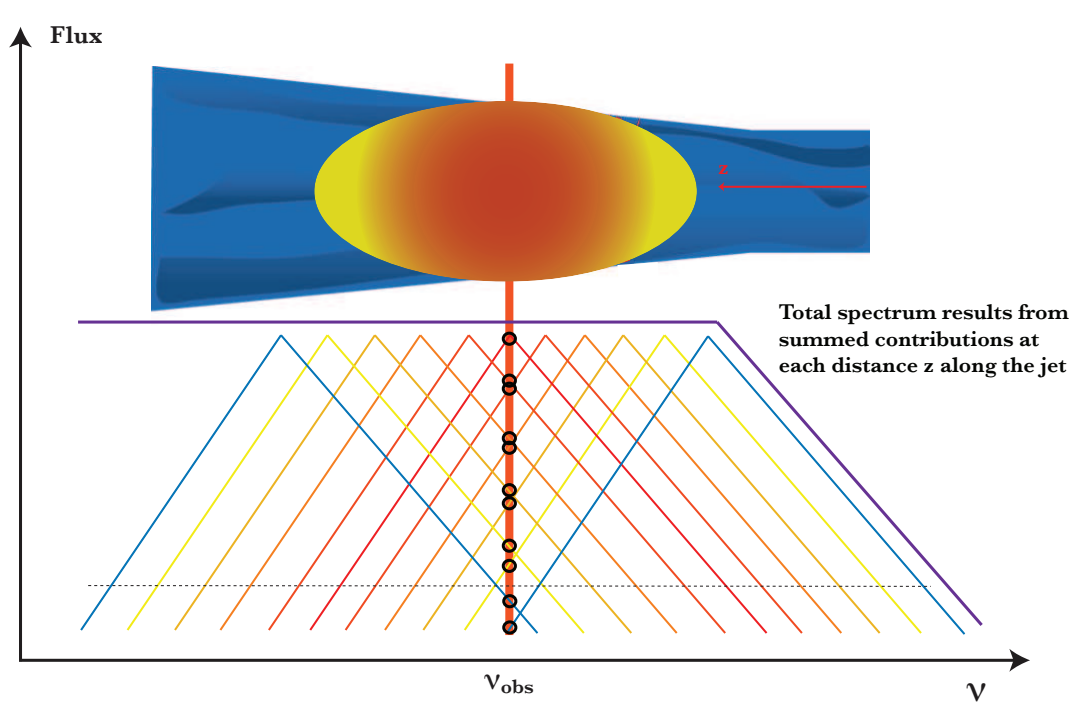

Fig. 2 Along the jet, the magnetic field decays. Thus, as the radiating particles propagate along the jet, the break frequencies presented in Figure 1 decay. While the emission from each slab has the same spectrum as presented in Figure 1 (right) peaking at $\nu_{S S A}$ (thin lines), when integrated over a spatially unresolved region along the jet, the observed radio spectrum is flat. The cartoon here is taken from Markoff (2010).

While these models show significant improvement in treating the dynamical properties of jets, the basic radiative mechanism discussed by Blandford and Konig] (1979) remains key to all of them. The radiative particles are assumed to have a power law distribution, and the peak of the emission is at $\nu_{S S A}$. The decrease of $\nu_{S S A}$ along the jet due to the decay of the magnetic field is the origin of the flat radio spectra.

An alternative approach was suggested by Pe'er and Casella (2009). Based on the idea of a single acceleration episode and the inclusion of particle cooling first proposed by Kaiser (2006), this model considers a scenario in which $\nu_{S S A}<\nu_{m}$. Thus, the peak of the emission from a given 'slab' is at $\nu_{m}$ (or $\nu_{c}$ ) rather than at $\nu_{S S A}$. As was shown in this work, as a result of the decaying magnetic field along the jet, the decay law of $\nu_{m}$ is identical to the decay law of $\nu_{S S A}$. Thus, a flat radio spectrum is naturally obtained in an analogous way to the Blandford and Konigl (1979) model, by integrating over emitting regions inside the jet (see Figure 3).

A conceptual difference between this model and the Blandford and Konigl (1979) model is that the former does not require a power law distribution of the accelerating electrons. As $\nu_{m}$ is one of the natural frequencies obtained from a Maxwellian distribution of radiating particles, a flat radio spectrum is obtained even if the acceleration process does not accelerate particles to a power law distribution. A second difference is that the change in particle distribution due to cooling is inherently considered. Thus, in a region of strong magnetic field the radiating particles rapidly cool, $\nu_{c}<\nu_{m}$, and the flux $F_{\nu} \propto \nu^{-1 / 2}$ (see Equation 7). This result is independent of the details of the acceleration process and the 


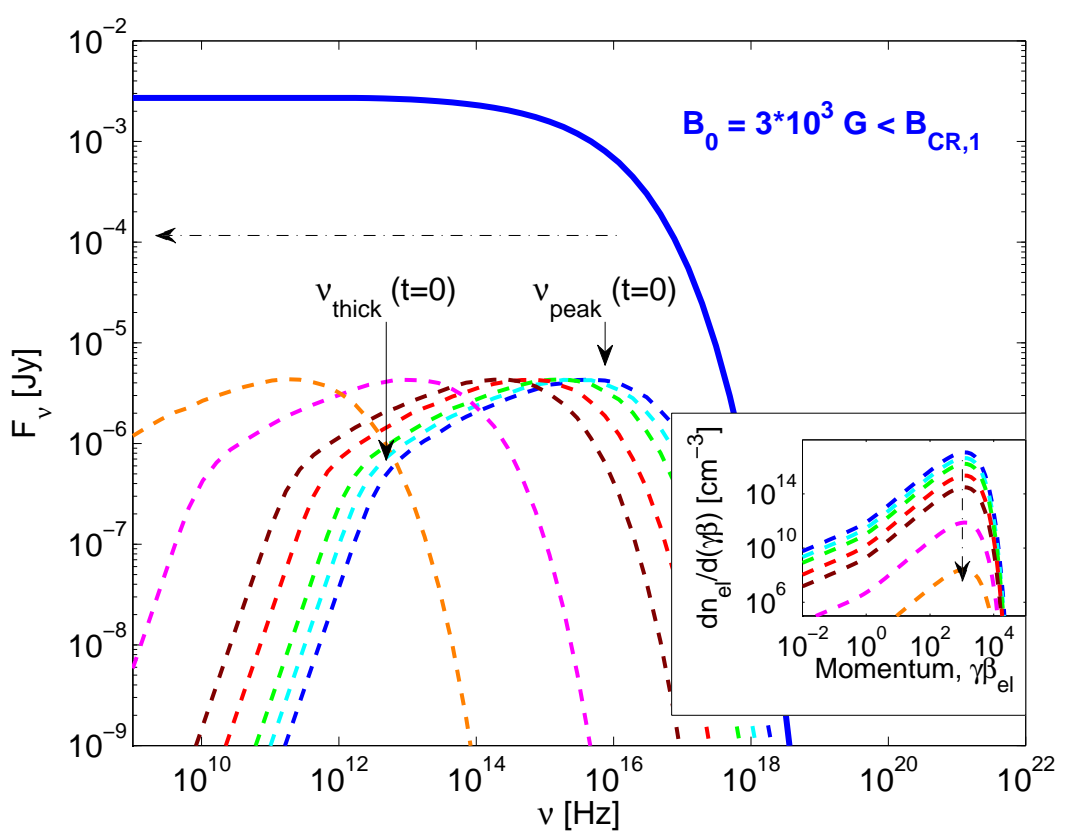

Fig. 3 The model of Pe'er and Casella (2009) provides an alternative way to explain flat radio spectra without the need for a power law distribution of the energetic particles. Emission from a Maxwellian distribution (dashed lines) is characterized by two break frequencies, $\nu_{m}$ (described in the figure as $\nu_{\text {peak }}$ ) and $\nu_{S S A}\left(\right.$ or $\nu_{\text {thick }}$ ). In the model considered, $\nu_{m}>\nu_{S S A}$, and thus the emission from a single slab peaks at $\nu_{m}$. As the particles propagate along the jet $\nu_{m}$ decays, and thus the integrated spectra resulting from a Maxwellian distribution of particles (inner set) is flat (thick blue line). The value of the magnetic field, $B=10^{3.5} \mathrm{G}$ is arbitrarily chosen for demonstration purposes only.

magnetic field structure. Finally, if the magnetic field is very strong, rapid cooling of the particles as they propagate along the jet leads to absorption of the emission peak, $\nu_{m}<\nu_{S S A}$ for emission that occurs above a certain radius. This, in turn, results in a suppression of the radio emission, as seen in several objects Casella and Pe'er (2009)].

One assumption common to all jet models is that the decay of the magnetic field leads to a decay of the characteristic frequencies along the jet. Thus, although unresolved, emission at low radio frequencies is expected from distant regions along the jet. Conversely, emission at higher energies - microwave, optical X- and $\gamma$-rays must originate from the inner parts of the jet, where the discrimination between the outflow (jet) and inflow (accretion) may be very difficult. As shown in Equation 5 . at least from a theoretical perspective synchrotron photons can be observed nearly to the $\mathrm{GeV}$-range, irrespective of the strength of the magnetic field. 
2.5 Fragmented outflow: emission from radio blobs

The flat radio emission is a natural outcome of emission in a power law decay of the magnetic field along the jet. Thus, the above discussed models are relevant when the outflow is continuous. However, fragmented ejection of material, in the form of radio "blobs" are frequently observed in both XRBs, such as GRS1915+15 Mirabel and Rodríguez (1994); Rodríguez and Mirabel (1999); Fender et al. (1999a); Miller-Jones et al. (2005)] as well as AGNs.

The different emission observed from these blobs with respect to their environment indicates different physical conditions inside the blobs. Particularly, it implies that the magnetic field and/or the distribution of radiating particles inside the blobs is different than outside of them. This is a natural consequence if the magnetic field and particle acceleration originate in shock waves, as discussed above. As the blobs propagate outward they expand. The expansion can be adiabatic, but not necessarily (it could be confined by, say an external magnetic field). Thus, one can deduce scaling laws for the evolution of the magnetic field and the particle distribution inside the expanding blobs. The basic model was suggested by van der Laan (1966), and extended by Hjellming and Johnston (1988); Atoyan and Aharonian (1999).

The key radiative model is similar to the Blandford and Konigl (1979) model, namely that particles are accelerated to a power law distribution, and the emission peaks at $\nu_{S S A}$. However, the scaling laws are different. The basic assumption is that particles do not enter or leave a blob, which is adiabatically expanding. Conservation of magnetic flux implies a decay of the magnetic field $B \propto L^{-2}$, and adiabatic cooling implies a decline in the particles' energy, $\gamma \propto L^{-1}$, where $L$ is the comoving size of the expanding blob. Since the emitted frequency $\nu \propto$ $B \gamma_{e l}^{2}$ (see Equation 11), one can derive the scaling law of the Lorentz factor of the radiating electrons at observed frequency $\nu$ to be $\gamma_{e l} \propto \nu^{1 / 2} B^{-1 / 2} \propto L$. For power law distribution $N(\gamma) d \gamma=k \gamma^{-S}$, the synchrotron flux then scales as $F_{\nu} \propto$ $k B^{(S+1) / 2} L^{3}$, where $k \propto L^{(-S+2)}$ [see van der Laan (1966); Rybicki and Lightman $(1979)]$.

These scaling laws thus give a testable prediction, $F_{\nu} \propto L^{-2 S} \sim t^{-2 S}$. When confronted with observations Rodriguez et al. (1995)], the observed decline is not as steep as the theoretical prediction. Thus, the simplified version of the theory needs to be adjusted. One natural possibility is that the expansion is not adiabatic. For example, reverse shock may play a significant role in determining the evolution of these blobs [R. Narayan, private communication].

\section{Compton scattering and the origin of the X-ray spectrum}

While there is a consensus that the radio spectrum originates from synchrotron radiation (although the full details of the process are uncertain), the origin of the X-ray and $\gamma$-ray emission is far more debatable. As shown above, synchrotron emission can extend up to hundreds of $\mathrm{MeV}$. However, at these energies, there are alternative sources of emission. In particular, Compton scattering of low energy photons by energetic electrons is a natural, alternative way to produce emission at these bands. Due to the larger cross section, even if hadrons (protons) contribute 
to the emission, their contribution to IC scattering is expected to be negligible compared to the electrons contribution.

Energetic electrons radiate their energy via both synchrotron radiation and IC scattering. The total power emitted by IC process is Rybicki and Lightman (1979)]

$$
\frac{P_{I C}}{P_{s y n}}=\frac{U_{p h}}{U_{B}}
$$

where $P_{s y n}$ is the synchrotron power, $U_{B}$ and $U_{p h}$ are the energy densities in the magnetic and photon fields, respectively. Thus, if $U_{p h}>U_{B}$, most of the electrons' energy is radiated by IC scattering rather than synchrotron. However, even if $U_{B}>U_{p h}$, it is still possible that IC scattering is the main source of emission at a given frequency band.

In understanding Compton scattering, the basic questions are therefore:

1. What is the origin and spectral distribution of the energetic electrons ? Obviously, this is a similar question to the one that lies in the heart of understanding synchrotron emission, as the same electrons radiate both synchrotron photons and IC photons.

2. What is the origin of the upscattered photon field ? Do these photons originate inside the jet (e.g., by synchrotron emission), or are they external to the jet (e.g., originating in the accretion flow or CMB) ?

3. Since electrons in the inner parts of the accretion flow are hot enough to emit in the X-ray band, is there a simple way to discriminate disk and jet emission by observing at this band?

The third question is particularly puzzling, and is the source of an intense debate. As discussed above, synchrotron emission from the inner parts of the jet, where the magnetic field is strongest, are expected to contribute to the observed flux at the $\mathrm{X}$ - and possibly also $\gamma$-ray frequencies. These regions are close to the inner parts of the inflow. Thus, discriminating between the inflow and jet as the sources of X-ray radiation is very challenging.

While IC emission from particles in AGN jets is well established, most works on X-ray emission in XRBs are focused on IC emission from the inner parts of the accretion flow. A few notable works are by Sunvaev and Titarchuk (1980); Haardt and Maraschi (1993); Titarchuk (1994); Magdziarz and Zdziarski (1995); Esin et al. (1997); Poutanen (1998); Cadolle Bel et al. (2006); Yuan et al. (2007), to name only a handful. As this is the subject of a separate chapter in this book, we will not discuss it here. I will point though, that there are various reasons to consider IC scattering from electrons inside the jets in XRBs. These include:

1. As the radio spectrum originates from synchrotron photons, energetic electrons exist in the jet. These electrons must upscatter low energy photons.

2. From a theoretical perspective, models in which the dominant contribution is IC emission from the inflow do not well connect to the need for strong magnetic fields required in leading jet-launching models discussed above (though a few recent accretion models may overcame this problem; see Ferreira et al. (2006); Fragile and Meier (2009); Bu et al. (2009); Oda et al. (2010); Petrucci et al. $(2010))$.

3. Detection of X-ray emitting blobs propagating outward in the inner regions of jets in several microquasars [Corbel et al. (2002)] indicate that part of the 
radiation in these objects is from the jet (or interaction of the jet with the ambient medium), and not all of it originates from the accretion flow.

4. Finally, the very high energy emission ( $\gtrsim 100 \mathrm{GeV})$ observed in several microquasars (or microquasar candidates) Aharonian et al. (2005); Albert et al. (2006, 2007b)] is difficult to explain in disk models.

\subsection{Origin of the seed photons.}

As particles acceleration were discussed in $\$ 2$ above, let us focus on the origin of the seed photons for IC scattering.

Synchrotron self Compton. A natural source of seed photons are the synchrotron photons emitted by the energetic electrons, namely SSC. As long as the scattering is in the Thomson regime, namely the energy of the upscattered photons is much less than the energy of the incoming electron, the outgoing photon energy is $\varepsilon_{\text {out }} \simeq 4 \gamma_{e l}^{2} \varepsilon_{\text {in }}$, where $\gamma_{e l}$ is the Lorentz factor of the electron [e.g., Rybicki and Lightman (1979)]. In this case, the spectral shape of SSC emission from a power law distribution of energetic electrons is similar to that of synchrotron emission discussed above [see, e.g., Sari and Esin (2001)]. It is characterized by four break frequencies. If $\nu_{m}<\nu_{c}$, the values of these break frequencies are $\nu_{S S A}^{I C} \simeq 4 \gamma_{m}^{2} \nu_{S S A}, \nu_{m}^{I C} \simeq 4 \gamma_{m}^{2} \nu_{m}$, and $\nu_{c}^{I C} \simeq 4 \gamma_{c}^{2} \nu_{c}$. If $\nu_{c}<\nu_{m}$, spectral break corresponding to the self absorption occurs at $\nu_{S S A}^{I C} \simeq 4 \gamma_{c}^{2} \nu_{S S A}$, while the energy of the other breaks is not changed.

Thus, for $\nu_{m}<\nu_{c}$ the peak of the IC spectrum is at $\nu_{m}^{I C}$ while for $\nu_{c}<\nu_{m}$ it is at $\nu_{c}^{I C}$, both naturally extend up and above the $\mathrm{MeV}$ range. The ratio of the IC and synchrotron energy fluxes is given by $\left(\nu F_{\nu}\right)_{\text {peak, IC }} /\left(\nu F_{\nu}\right)_{\text {peak,syn }}=Y$, where $Y=(4 / 3) \gamma_{e l}^{2} \tau$ is the Compton $Y$ parameter and $\tau$ is the optical depth.

In AGN jets two distinct, broad spectra components are observed. The low energy component is peaking at the sub-mm to IR regime (in FSRQ) or in the UV / X-rays (in high-frequency peaked BL Lacs, or HBLs). The high energy component peaks at the MeV energies in FSRQs and GeV energies in HBLs [e.g., Fossati et al. (1998); Donato et al. (2001); Sambruna et al. (2004); Levinson (2006) and references therein]. An example of this spectra is presented in Figure 4, taken from Sikora et al. (1994). It is therefore natural to attribute the peak at the radio band to synchrotron emission, while that at the X-ray band to IC, as is done by many authors Konigl (1981); Marscher and Gear (1985); Maraschi et al. (1992); Hartman et al. (2001); Finke et al. (2008); Ghisellini et al. (2009, 2010) and many more]. In fact, lacking good theoretical knowledge of the electron number density, hence of the optical depth, very often it is being determined by fitting the ratio of IC peak flux to the synchrotron peak flux. In recent years, similar fitting was done to the X-ray spectra in XRBs [e.g., Gupta et al. (2006); Gallo et al. (2007); Migliari et al. (2007); Maitra et al. (2009)].

External seed photons. In addition to SSC, there are other sources of seed photons. As the spectral shape of the resulting IC emission depends on the spectral shape of the incoming photons, broad band fitting of the spectra are required to determine which is the dominant field. One natural source is the photon field created by the accretion disk Begelman and Sikora (1987); Dermer et al. (1992); Dermer and Schlickeiser (1993); Blandford and Levinson (1995)]. In XRBs, pho- 


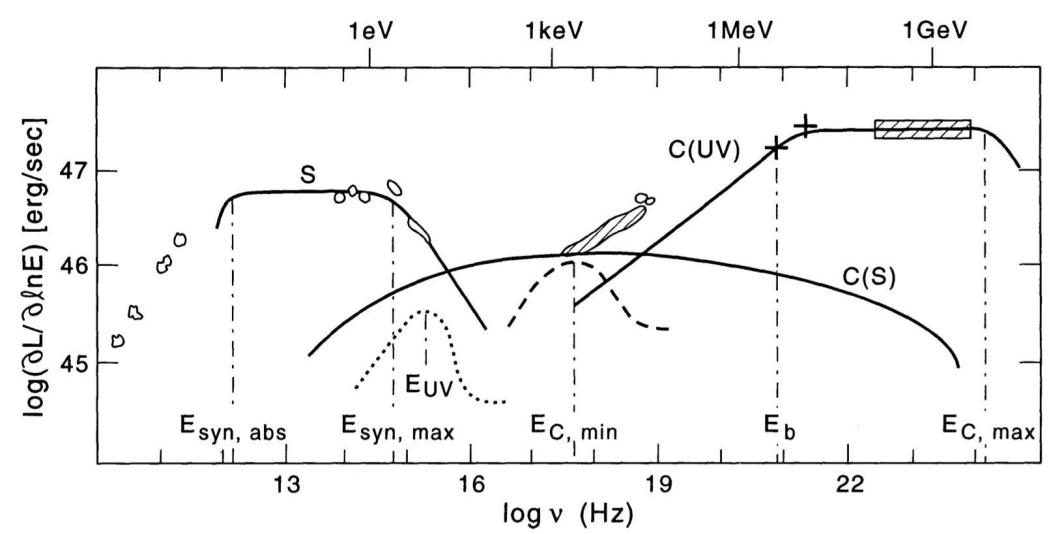

Fig. 4 Broad band spectrum of the AGN 3C 279, taken from Sikora et al. (1994). Solid lines mark the fitted model spectral components: (S) - synchrotron, C(S) - SSC, C(UV) Comptonization of diffusive UV radiation. For further details as well as references about the data points, see Sikora et al. (1994).

tons from the companion star can also serve as seed photons for IC scattering Dermer and Böttcher (2006)].

Alternative source of photons is reprocessing of disk emission by the surrounding material, such as the broad emission line region in AGNs [Sikora et al. (1994); Dermer et al. (1997); see Figure 4. Additional suggestions for seed photons include reprocessing of the synchrotron emitted photons from the jet itself by the surrounding medium before being IC scattered [Ghisellini and Madau (1996)], infrared emission from circumnuclear dust [Błażejowski et al. (2000)] or synchrotron radiation from other regions along the jet itself Georganopoulos and Kazanas (2003)]. Clearly, these models require additional assumptions about the environment and/or the material that acts to reprocess the original emission. The addition of degrees of freedom with respect to the synchrotron-SSC model enables much better fits to existing broad band data at the price of more complex modeling of the environment.

In nearby objects such as Cen $\mathrm{A}$, the extended giant radio lobes can be spatially resolved. Two distinguished spectral components, one at the radio band Hardcastle et al. (2009)] and one at the X-ray and/or $\gamma$-rays Feigelson et al. (1995); Kataoka and Stawarz (2005); Croston et al. (2005); Abdo et al. (2010)] are detected in these lobes. While the radio spectrum is naturally attributed to synchrotron emission, at such large distances from the core (typically hundreds of $\mathrm{kpc}$ ) it is insufficient to provide enough photons to explain the X-and $\gamma$-ray flux observed. Instead, this is attributed to IC emission of the cosmic microwave background (CMB) or extra-galactic background (EBL) light Tavecchio et al. (2000); Celotti et al. (2001)], whos' spectra are well known Georganopoulos et al. (2008); Finke et al. (2010)]. This scenario has a great advantage, as it enables decoupling of the electrons distribution and the magnetic field. First, the electron distribution is inferred from the IC spectra and the known seed photon field, and at a second step the magnetic field strength is inferred from the synchrotron spectrum. This separation thus enables to infer the values of the magnetic fields in these regimes, which is found to be close to equipartition. Since these values are much larger than 
can be achieved by Poynting-flux conservations from the core, as well as higher than the (compressed) external field, these results point towards magnetic field generation in shock waves, as discussed above. Moreover, analyzing the spectra enables to show that the conditions in these lobes enable acceleration of particles to ultra-high energies Pe'er and Loeb (2012)].

\subsection{Separation between disk and jet photons}

As much as inferring the origin of seed photons at large distances along the jet is not easy, close to the jet base the situation is far more complicated. Separating jet-based emission models from disk-based emission models is a very difficult task, as both disk-based and jet-based models can produce good fits to the data Markoff et al. (2005)].

On the one hand, there are some indirect evidence based on correlation between emission at the X-ray band and lower energy bands (radio, IR and optic) for jetdominated X-ray emission Yuan et al. (2009); Russell et al. (2010)]. This interpretation is strengthen by extrapolation of the spectral energy distribution (SED) above the turn over at mid- IR bands Gandhi et al. (2011); Rahoui et al. (2011, 2012); Russell et al. (2013); Corbel et al. (2013)]. An independent support comes from polarization measurements by INTEGRAL satellite [Laurent et al. (2011); Jourdain et al. (2012)], which show strong polarization above $400 \mathrm{keV}$ in Cyg X-1, hinting towards jet origin in these energies.

In spite of these indications, it should be stressed that as of today, disk-based models for the X-ray emission are much more developed, and are favored by a central part of the community. As these models are thoroughly discussed in other chapters of this book, I will only briefly mention some aspects here. In these models, many of the X-ray properties are explained by Comptonization in hot accretion flows, including detailed X-ray spectral shape [e.g., Sobolewska et al. (2012); Qiao and Liu (2013)], spectral evolution during state transition Del Santo et al. (2013)], and many timing properties of X-ray variability Kotov et al. (2001); Ingram and Done (2011, 2012)]. Moreover, detailed fitting of X-ray spectra with the jet models require, in some cases, optical depth of $\tau \sim 2-3$ Malzac and Belmont (2009); Poutanen and Vurm (2009); Droulans et al. (2010)], which put strong constraints on the jet kinetic power Malzac et al. (2009)]. Further critical discussions about jet vs. disk models can be found in Poutanen and Zdziarski (2003); Zdziarski et al. (2003); Maccarone (2005); Veledina et al. (2013) as well in other chapters of this book.

One difference between the two scenarios is that while electrons in the inflow are expected to be continuously heated as they spiral in, it is possible that once they enter the jet region they are no longer heated. As they propagate outwards inside the jet, both the radiation field and the magnetic field decay, and thus cooling of the electrons is suppressed. During their initial propagation outward, they do though radiatively cool very rapidly. For rapidly cooling electrons, both synchrotron emission discussed above and Compton scattering produce the same spectrum: $F_{\nu} \propto \nu^{-1 / 2}$ in the range $\nu_{c}<\nu<\nu_{m}$. This spectrum is consistent with the X-ray spectra observed in many outbursts in XRBs [e.g., Hynes et al. (2000); 


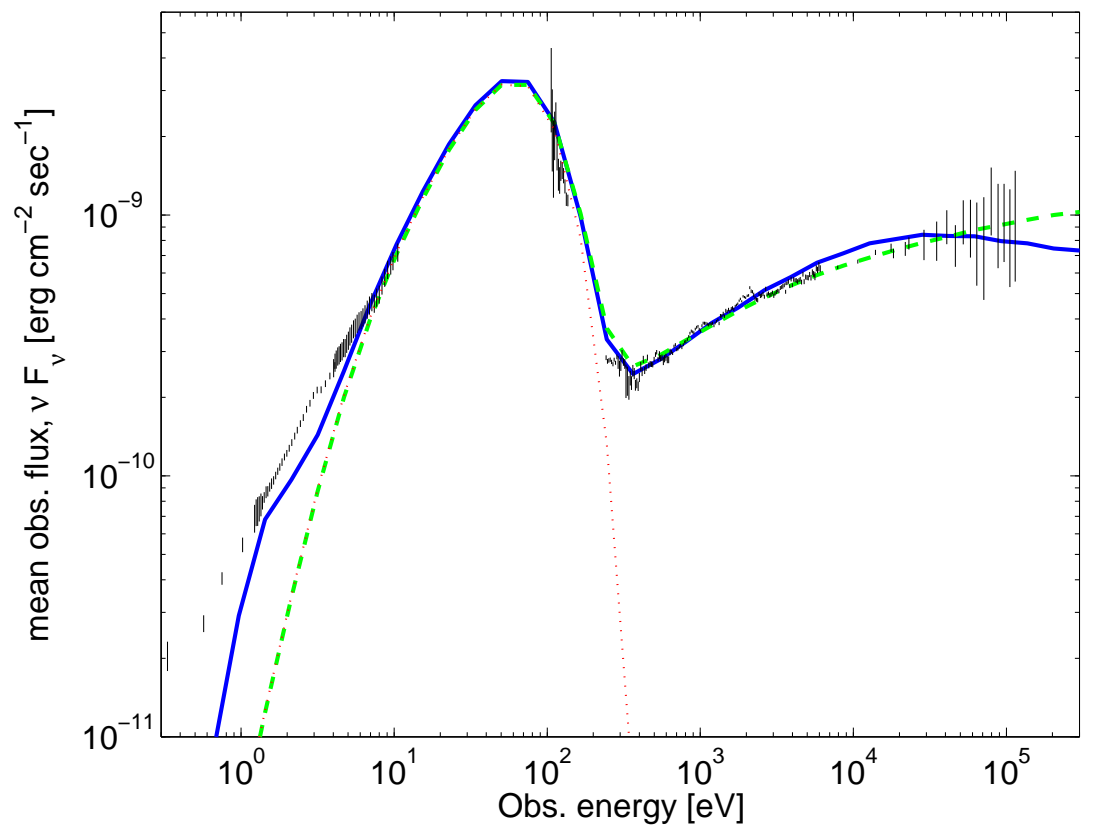

Fig. 5 Fitting the 2000 outburst of XTE J1118+480 (taken from Pe'er and Markoff (2012)). The solid (blue) curve represent a model in which synchrotron emission is the main source of radiation, while the dashed (green) represents an IC-dominated model. Both models provide good fits to the data, and are consistent with emission from electrons in the inner part of the jet. For further details see Pe'er and Markoff (2012).

Esin et al. (2001); Homan et al. (2005); Joinet et al. (2008)] ]1. Thus, by identifying the break frequencies seen in the spectra with $\nu_{m}$ and $\nu_{c}$, it is possible to constraint the physical parameters from the emitting region. In particular, this analysis may enable to discriminate between emission from the inner parts of the jet (in which the electrons reside only a short time), and emission from the inflow, which is expected to last over a longer period, during the spiral-in. Such an analysis was carried by Pe'er and Markoff (2012), and one of its results is presented in Figure $\left[5^{2}\right.$

\section{Hadronic contribution to the high energy spectra}

The uncertainty in the nature of the acceleration process implies that protons may be accelerated as well inside the jets. Once accelerated to high energies, protons contribute to the observed spectrum. Although synchrotron emission and Compton scattering are suppressed with respect to emission from leptons due to the much

\footnotetext{
1 Jet dominated models are expected in XRBs during the low/ hard state, where $L \sim$ $1 \% L_{E d d}$. At higher luminosities, disk contribution is expected, and the spectral slope varies; the luminosity-dependence of the spectral index can be found in $\mathrm{Wu}$ and $\mathrm{Gu}$ (2008).

2 Data taken from McClintock et al. (2001); Note, though, that a different analysis of BeppoSAX [Frontera et al. (2001)] and Chandra Reis et al. (2009)] data done in the context of disk models, resulted in a somewhat softer slope below $2 \mathrm{keV}$.
} 
smaller cross section, protons may still have a significant contribution to the high energy emission. First, if the acceleration process acts in such a way that most of the energy is deposited in accelerated protons, it is possible that synchrotron emission from these protons have a significant contribution to the high energy (Xand $\gamma$-ray) flux Aharonian (2000)]. Second, energetic protons can deposit their energy by photo-meson production, $p \gamma \rightarrow n+\pi^{+}, p+\pi^{0}$ Mannheim and Biermann (1992); Mannheim (1993)]. The created $\pi$ mesons are unstable; the $\pi^{+}$can radiate synchrotron emission before decaying into $\mu^{+}+\nu_{\mu} \rightarrow e^{+}+\nu_{e}+\bar{\nu}_{e}+\nu_{\mu}$, while the $\pi^{0}$ decays into a pair of energetic photons. These particles thus produce a high energy electromagnetic cascade, as the created photons are energetic enough to produce a pair of electron-positron, $\gamma+\gamma \rightarrow e^{ \pm}$. Emission from these secondaries may thus be responsible for the high energy (up to TeV) emission seen in AGNs (blazars) Rachen and Mészáros (1998); Mücke et al. (2003); Murase et al. (2012)], as well as in GRBs [Pe'er and Waxman (2004, 2005a)] and XRBs [Romero et al. (2005)].

In addition to photomeson production, protons can interact with photons by photopair production $\left(p+\gamma \rightarrow p+e^{ \pm}\right)$, and with other protons through protonproton (pp) collisions, producing pions and Kaons [Koers et al. (2006)]. The rate of these interactions depend of course on the ambient photon field, as well as the uncertain distribution of the energetic protons. Combined leptonic/hadronic models that explain the radio emission in AGNs and XRBs as due to synchrotron radiation from electrons and the high energy (up to TeV) emission as due to hadronicoriginated cascade exist [e.g., Vila and Romero (2010); see Figure 6]. Although, as can be seen from Figure 6, these models are still lagging behind disk models, and can thus currently can still only provide approximate fits to the observed spectra. These models suffer two main drawback: First, the inherent uncertainty in the knowledge of the accelerated proton distribution. Second, calculating the evolution of the high energy electromagnetic cascade is extremely difficult, due to the non-linearity of the process, and the fact that it is very rapid, namely, many orders of magnitude shorter than the dynamical time. Thus, it is numerically challenging. While in recent years models of cascade evolution in GRB environment exist Pe'er and Waxman (2005b)], this field is still at its infancy.

An interesting consequence of hadronic models, is that if indeed protons are accelerated to high energies, among the secondaries produced are high energy neutrinos [for a review about neutrino production in AGN jets see Gaisser et al. (1995); for neutrino production in XRB jets, see Levinson and Waxman (2001); Christiansen et al. (2006); Zhang et al. (2010)] . Thus, such neutrinos - if detected - would be a direct proof of proton acceleration in these environments.

\section{Temporal information}

5.1 XRBs: temporal correlation between different spectral bands

Although the basic radiative processes are well known, the emitted spectra from jets are very complex, due to the complex nature of these systems. Emission originates from both the accretion flow, different regions along the jet where the physical conditions vary, as well as external photons that can be reprocessed (IC scattered) from particles along the jet. The physical conditions along the jet, such as the magnetic field and particle distribution and their connection to the physical 


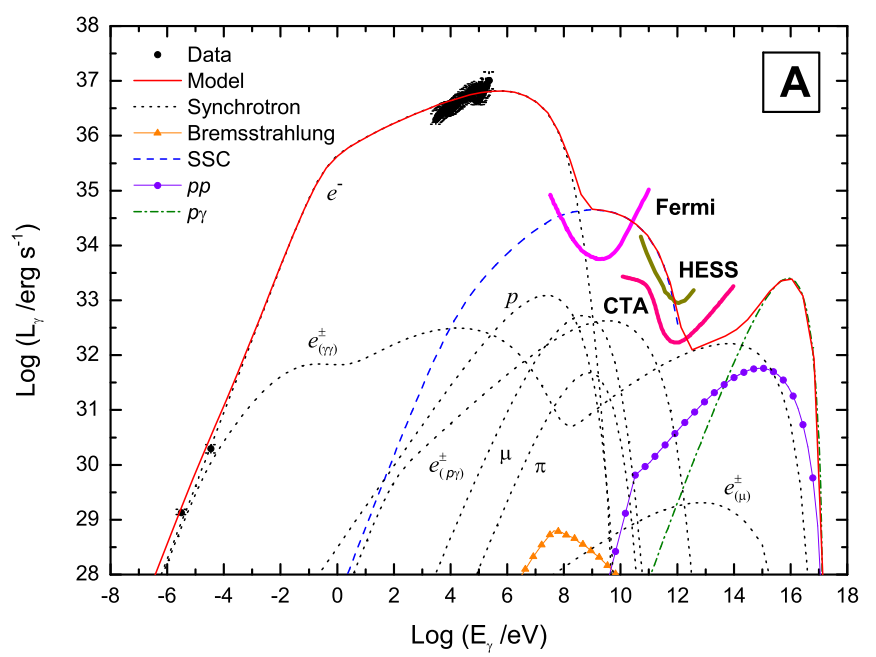

Fig. 6 Fitting the broad band spectra of the 1997 outburst of GX 339-4 by a leptonic/hadronic model of Vila and Romero (2010). Contribution from the various processes are marked: generally, protons contribution is expected mainly at high energies, X- and $\gamma$-rays. These fits are used to infer the uncertain values of the magnetic field as well as the electron/proton distribution. For details see Vila and Romero (2010).

conditions in the inner parts of the inflow are uncertain. It is thus not surprising that the observed spectra can be interpreted in more than one way, and that plethora of models aimed at explaining the broad band spectra exist. An in depth discussion in some of the models appear in the chapters authored by Fender, Gallo, Casella and Körding in this book.

Thus, in order to obtain a full picture additional information is needed. In XRBs, a natural source of information is temporal analysis, since the emission pattern conveniently changes over time scale of $\lesssim$ months. As the emission changes with time, correlation between emission from the inflow and the jet at different times (the different "states") is established. Such correlation is the switch off of jet radio emission in the high/soft state Tananbaum et al. (1972); Fender et al. $(1999 \mathrm{~b})]$. Others are the correlation found between the X-ray luminosity and the radio luminosity Hannikainen et al. (1998); Corbel et al. (2000, 2003); Gallo et al. (2003)], which are found to scale as $L_{R} \propto L_{X}^{0.7}$. However, recently, it was shown that the system H1743-322 follow a different correlation, $L_{R} \propto L_{X}^{1.4}$ Coriat et al. (2011); Gallo et al. (2012)]. Other correlations are found at different wavebands: between the X and near infrared (NIR) Russell et al. (2006); Coriat et al. (2009); Casella et al. (2010)], and radio - optic and X-rays [Kanbach et al. (2001); Gandhi et al. (2010); Cadolle Bel et al. (2011)].

The wealth of emitting zones and radiative processes enables to interpret the observed correlations in various ways. One type of models explore the obvious (yet uncertain) connection between the properties of the inner parts of the accretion flow and the jet [e.g., Markoff et al. (2003); Heinz and Sunyaev (2003)]. Other 
ideas include the obvious connection between the synchrotron radiation and IC scattering by the same population of electrons Giannios (2005); Veledina et al. (2011); see details in the chapter by Poutannen in this book], as well as correlation between (synchrotron) emission by the same electrons as they propagate along the jet thereby occupying different regions in the jet at different times Casella et al. $(2010)]$.

Existence of different emission zones reflects the complex internal dynamics of the outflow. For non-steady outflow, shock waves naturally develop when two "blobs", or shells of plasma collide. This happens once the ejection of a slower plasma blob is followed by ejection of faster moving one. Once the blobs collide, two shock waves are formed, propagating into both plasmas. By heating (and possibly accelerating to high energies) the particles, these shock waves are the initial source of radiation. This scenario was invoked to explain the complex lightcurve seen during GRB prompt emission Rees and Meszaros (1994); Daigne and Mochkovitch (1998)]. In recent years, similar ideas were studied in the context of emission from XRBs Kaiser et al. (2000); Jamil et al. (2010); Malzad (2013)], and TDEs Giannios and Metzger (2011); although a structured jet model was suggested by Liu et al. (2012)].

This idea, though, is incomplete: currently, the internal shock model is lacking a predictive power about the radii at which the collisions, hence the energy dissipation takes place - these are determined by the initial conditions. Thus, overall, my personal opinion is that understanding the nature of the correlations observed is at its infancy, and that this field is a very promising path to take. Future models will inevitably combine both dynamical models and radiative models, which will mature in the coming years.

\subsection{Flaring activities in AGNs}

In AGNs (blazars), flaring activity is observed in the X-and $\gamma$-rays up to the highest energies, at the $\mathrm{TeV}$ band. This is often observed on a very short time scales, of the order of hours and in some cases even minutes Kniffen et al. (1993); Buckley et al. (1996); Aharonian et al. (2007); Albert et al. (2007a); Aleksić et al. (2011)]. Radio observations showed that radio outburst seem to follow the $\gamma$-ray flares Reich et al. (1993); Zhang et al. (1994)]. While significant variability in the optical band is observed as well, its correlation with the variability in the $\gamma$-band is not fully clear Wehrle et al. (1998); Palma et al. (2011)].

The main implication of this rapid variability in the flux is constraining the size of the emitting region and the bulk motion Lorentz factor. An observed variability time $\Delta t^{\mathrm{ob}}$ implies that the size of the emitting region cannot exceed

$$
r \leq r_{\mathrm{var}} \approx \frac{\Gamma c \Delta t^{\mathrm{ob}} \mathcal{D}}{1+z}
$$

where $z$ is the redshift, and $\Gamma$ is the Lorentz factor associated with the bulk motion Ghisellini and Madau (1996)]. On the other hand, the fact that TeV photons are observed implies that the optical depth to pair production with the low energy photons in the plasma cannot exceed unity. Thus, the emitting region cannot be too compact. Combined together, these two constraints imply high bulk Lorentz factor (e.g., in PKS 2155-304, $\Gamma \gtrsim 50$ was inferred by Begelman et al. (2008)). The 
exact value of the constraint on the emitting region thus depend on the variability time, as well as the photon field. The variability itself reflects changing conditions within the outflow, e.g., due to the existence of internal shock waves Spada et al. (2001)]. The fact that the constraints found on $\Gamma$ in PKS 2155-40 were found to be inconsistent with direct measurements, have led Giannios et al. (2009) to suggest a jet within a jet model for the high energy emission. In a more general form, this can be viewed as an indication for an internal structure within the jets.

\section{Jet power}

Estimating the total deposited energy (or power) in astronomical jets is a very tricky task. The complexity of the problem is most easily understood if one considers the different episodes of energy transfer in these systems. First, there is the kinetic energy associated with the bulk motion of particles inside the jet. Naturally, this is some fraction of the gravitational energy of the inflowing material in the accretion disk. Theoretical determination of this fraction is possible only after the theory of jet production is fully understood. Until then, it can only be estimated from observations.

The observed radiation, in turn, reveals only a small fraction of this energy. Following jet launching, the second energy transfer occurs at a certain location(s) along the jet, where particles are accelerated to high energies. This acceleration must occur on the expense of (part of) the bulk motion kinetic energy, but possibly also due to magnetic reconnection - in which case it is at the expense of magnetic energy. Finally, the accelerated particles radiate some fraction of their energy as photons, producing the observed signal. Thus, direct observation of the photon signal reveals only an unknown fraction - likely a small fraction, of the kinetic energy initially given to the particles inside the jets.

Estimating the kinetic jet power is thus difficult, and rely on several assumptions. For example, Rawlings and Saunders (1991) estimated the average kinetic power of jets in blazars by dividing the total energy stored in the form of electrons and the magnetic field energy in the radio lobes (as deduced from synchrotron theory and the equipartition assumption) by the lobe age, which was computed from spectral aging or expansion velocity arguments. Similarly, Celotti and Fabian (1993) estimated the jet power of blazer jets using the framework of the standard synchrotron self-Compton theory. As explained above, these works suffer from substantial uncertainties, due to the various underlying assumptions needed.

In an alternative approach, Allen et al. (2006); Balmaverde et al. (2008) estimated the jet kinetic power by estimating the mechanical work, $P d V$ required to inflate the observed giant X-ray cavities. Since here too there are uncertainties in estimating the size of these cavities, these translate into uncertainties in the jet power. This will further be discussed in the chapter of this book authored by Heinz.

These works found a strong correlation between the estimated jet power and the disk luminosity. Such a correlation is expected in the leading mechanisms for jet production. As material from the jet originates from the disk, such correlations are of no surprise. Additional clue may come from a correlation between the jet power (as estimated from the radio flux) and spin of the black hole, as recently reported Narayan and McClintock (2012); Steiner et al. (2013)]. While this result 
is still debatable (see Russell et al. (2013)), if confirmed it may serve as a strong clue for the mechanism that launches jets in nature. See further discussions in the chapters by McClintock, Narayan, Fender and Gallo in this book.

Thus, while various models that estimate the kinetic power of jets exist, they all suffer from uncertainties, caused both by uncertainties in the measurements, and also by the need to rely on uncertain emission models. I thus view this subject as one which is far from being matured, and will be further developed in the near future.

\section{Summary and conclusions}

In this chapter, I reviewed some of the basic radiative mechanisms that produce the broad band emission seen in astronomical jets. Due to the broad nature of this subject, I focused on XRBs and AGNs (mainly blazars). The main radiative processes considered are synchrotron emission, SSC, Compton scattering of external, or reprocessed photons, and hadronic contribution, via proton-synchrotron emission and electromagnetic cascade caused by secondaries produced by protonphoton (and to a lesser extent, proton-proton) interactions.

Although each of these processes is well understood, the changing conditions inside the jets lead to complex observed spectra. This leads to the fact that inspite a wealth of broad-band data, no single model is commonly accepted. On the contrary, as discussed here, the same data can be interpreted in more than one way. Thus, the main "take away" massage from this chapter, is that modeling emission from jets is one of the most challenging tasks.

The questions that need to be addressed when studying emission from jets extend far beyond the realm of the radiative processes involved, and require addressing questions in basic physics and astronomy. Broadly speaking, in order to fully understand the emission, one needs to understand:

1. The connection between disk and jet, and the mechanism that leads to jet launching.

2. The varying physical conditions in different regions inside the jet, such as the magnetic field along the jet.

3. The jet composition that governs the contribution of leptons and hadrons to the observed spectra.

4. The nature and details of the acceleration mechanisms that determine the energy distribution of energetic particles in different parts of the jet.

5. The internal (synchrotron) and external (accretion disk, companion star, CMB, etc.) photon fields that serve as seed photons to scattering by jet material.

6 . The geometry of the jets, including velocity profile and its angle towards the observer, that determine the different scattered field, as well as the Doppler boost.

7. The dynamics of material inside the jet, that determines the spatial distribution of the radiating particles and their temporal evolution.

Addressing each of these questions is a task so challenging by itself, that despite decades of research and numerous works (unfortunately, only very few could be mentioned here) we still have only clues, but no definite answer to any of them. Moreover, these questions, while can be addressed separately, should be addressed 
in the context of the different environments in which jets are observed - XRBs, AGNs, GRBs and recently also TDEs. Thus, full answer to all these questions is not expected any time in the near future. However, the wealth of current and future data - both spectral, temporal and spatial data, ensures that there is plenty of room for new ideas in the coming years.

Acknowledgements I would like to thank Paul Callanan, Piergiorgio Casella, Amir Levinson and Abraham Loeb for providing useful comments on this manuscript.

\section{References}

A.A. Abdo, M. Ackermann, M. Ajello, W.B. Atwood, L. Baldini, J. Ballet, G. Barbiellini, D. Bastieri, B.M. Baughman, K. Bechtol, et. al., Fermi Gamma-Ray Imaging of a Radio Galaxy. Science 328, 725 (2010). doi:10.1126/science.1184656

A. Achterberg, Y.A. Gallant, J.G. Kirk, A.W. Guthmann, Particle acceleration by ultrarelativistic shocks: theory and simulations. Mon. Not. R. Astron. Soc. 328, 393-408 (2001). doi:10.1046/j.1365-8711.2001.04851.x

F.A. Aharonian, TeV gamma rays from BL Lac objects due to synchrotron radiation of extremely high energy protons. New A. 5, 377-395 (2000). doi:10.1016/S1384-1076(00)000397

F. Aharonian, A.G. Akhperjanian, K.-M. Aye, A.R. Bazer-Bachi, M. Beilicke, W. Benbow, D. Berge, P. Berghaus, et. al., Discovery of Very High Energy Gamma Rays Associated with an X-ray Binary. Science 309, 746-749 (2005). doi:10.1126/science.1113764

F. Aharonian, A.G. Akhperjanian, A.R. Bazer-Bachi, B. Behera, M. Beilicke, W. Benbow, D. Berge, K. Bernlöhr, et. al., An Exceptional Very High Energy Gamma-Ray Flare of PKS 2155-304. Astrophys. J. 664, 71-74 (2007). doi:10.1086/520635

J. Albert, E. Aliu, H. Anderhub, P. Antoranz, A. Armada, M. Asensio, C. Baixeras, J.A. Barrio, et. al., Variable Very-High-Energy Gamma-Ray Emission from the Microquasar LS I +61 303. Science 312, 1771-1773 (2006). doi:10.1126/science.1128177

J. Albert, E. Aliu, H. Anderhub, P. Antoranz, A. Armada, C. Baixeras, J.A. Barrio, H. Bartko, et. al., Variable Very High Energy $\gamma$-Ray Emission from Markarian 501. Astrophys. J. 669, 862-883 (2007a). doi:10.1086/521382

J. Albert, E. Aliu, H. Anderhub, P. Antoranz, A. Armada, C. Baixeras, J.A. Barrio, H. Bartko, et. al., Very High Energy Gamma-Ray Radiation from the Stellar Mass Black Hole Binary Cygnus X-1. Astrophys. J. 665, 51-54 (2007b). doi:10.1086/521145

J. Aleksić, L.A. Antonelli, P. Antoranz, M. Backes, J.A. Barrio, D. Bastieri, J. Becerra González, W. Bednarek, et. al., MAGIC Discovery of Very High Energy Emission from the FSRQ PKS 1222+21. Astrophys. J. 730, 8 (2011). doi:10.1088/2041-8205/730/1/L8

S.W. Allen, R.J.H. Dunn, A.C. Fabian, G.B. Taylor, C.S. Reynolds, The relation between accretion rate and jet power in X-ray luminous elliptical galaxies. Mon. Not. R. Astron. Soc. 372, 21-30 (2006). doi:10.1111/j.1365-2966.2006.10778.x

H. Alvarez, J. Aparici, J. May, P. Reich, The radio continuum spectrum of Centaurus A's large-scale components. Astron. Astrophys. 355, 863-872 (2000)

A.M. Atoyan, F.A. Aharonian, Modelling of the non-thermal flares in the Galactic microquasar GRS 1915+105. Mon. Not. R. Astron. Soc. 302, 253-276 (1999). doi:10.1046/j.13658711.1999.02172.x

M. Axelsson, L. Baldini, G. Barbiellini, M.G. Baring, R. Bellazzini, J. Bregeon, M. Brigida, P. Bruel, R. Buehler, G.A. Caliandro, R.A. Cameron, P.A. Caraveo, C. Cecchi, Chaves, et. al., GRB110721A: An Extreme Peak Energy and Signatures of the Photosphere. Astrophys. J. 757, 31 (2012). doi:10.1088/2041-8205/757/2/L31

B. Balmaverde, R.D. Baldi, A. Capetti, The accretion mechanism in low-power radio galaxies. Astron. Astrophys. 486, 119-130 (2008). doi:10.1051/0004-6361:200809810

M.C. Begelman, M. Sikora, Inverse Compton scattering of ambient radiation by a cold relativistic jet - A source of beamed, polarized continuum in blazars? Astrophys. J. 322, 650-661 (1987). doi:10.1086/165760

M.C. Begelman, R.D. Blandford, M.J. Rees, Theory of extragalactic radio sources. Reviews of Modern Physics 56, 255-351 (1984). doi:10.1103/RevModPhys.56.255 
M.C. Begelman, A.C. Fabian, M.J. Rees, Implications of very rapid TeV variability in blazars. Mon. Not. R. Astron. Soc. 384, 19-23 (2008). doi:10.1111/j.1745-3933.2007.00413.x

A.R. Bell, The acceleration of cosmic rays in shock fronts. I. Mon. Not. R. Astron. Soc. 182, 147-156 (1978)

R. Blandford, D. Eichler, Particle acceleration at astrophysical shocks: A theory of cosmic ray origin. Phys. Rep. 154, 1-75 (1987). doi:10.1016/0370-1573(87)90134-7

R.D. Blandford, Accretion disc electrodynamics - A model for double radio sources. Mon. Not. R. Astron. Soc. 176, 465-481 (1976)

R.D. Blandford, A. Konigl, Relativistic jets as compact radio sources. Astrophys. J. 232, 34-48 (1979). doi:10.1086/157262

R.D. Blandford, A. Levinson, Pair cascades in extragalactic jets. 1: Gamma rays. Astrophys. J. 441, 79-95 (1995). doi:10.1086/175338

R.D. Blandford, C.F. McKee, Fluid dynamics of relativistic blast waves. Physics of Fluids 19, 1130-1138 (1976). doi:10.1063/1.861619

R.D. Blandford, C.F. McKee, Radiation from relativistic blast waves in quasars and active galactic nuclei. Mon. Not. R. Astron. Soc. 180, 343-371 (1977)

R.D. Blandford, J.P. Ostriker, Particle acceleration by astrophysical shocks. Astrophys. J. 221, 29-32 (1978). doi:10.1086/182658

R.D. Blandford, D.G. Payne, Hydromagnetic flows from accretion discs and the production of radio jets. Mon. Not. R. Astron. Soc. 199, 883-903 (1982)

R.D. Blandford, R.L. Znajek, Electromagnetic extraction of energy from Kerr black holes. Mon. Not. R. Astron. Soc. 179, 433-456 (1977)

M. Błażejowski, M. Sikora, R. Moderski, G.M. Madejski, Comptonization of Infrared Radiation from Hot Dust by Relativistic Jets in Quasars. Astrophys. J. 545, 107-116 (2000). doi: $10.1086 / 317791$

G.R. Blumenthal, R.J. Gould, Bremsstrahlung, Synchrotron Radiation, and Compton Scattering of High-Energy Electrons Traversing Dilute Gases. Reviews of Modern Physics 42, 237-271 (1970). doi:10.1103/RevModPhys.42.237

V. Bosch-Ramon, G.E. Romero, J.M. Paredes, A broadband leptonic model for gammaray emitting microquasars. Astron. Astrophys. 447, 263-276 (2006). doi:10.1051/00046361:20053633

D.-F. Bu, F. Yuan, F.-G. Xie, Self-similar solution of hot accretion flows with ordered magnetic field and outflow. Mon. Not. R. Astron. Soc. 392, 325-331 (2009). doi:10.1111/j.13652966.2008.14047.x

J.H. Buckley, C.W. Akerlof, S. Biller, D.A. Carter-Lewis, M. Catanese, M.F. Cawley, V. Connaughton, D.J. Fegan, J.P. Finley, J. Gaidos, A.M. Hillas, J.F. Kartje, A. Koenigl, F. Krennrich, R.C. Lamb, R. Lessard, D.J. Macomb, J.R. Mattox, J.E. McEnery, G. Mohanty, J. Quinn, A.J. Rodgers, H.J. Rose, M.S. Schubnel, G. Sembroski, P.S. Smith, T.C. Weekes, C. Wilson, J. Zweerink, Gamma-Ray Variability of the BL Lacertae Object Markarian 421. Astrophys. J. 472, 9 (1996). doi:10.1086/310352

D.N. Burrows, J.A. Kennea, G. Ghisellini, V. Mangano, B. Zhang, K.L. Page, M. Eracleous, P. Romano, T. Sakamoto, A.D. Falcone, J.P. Osborne, S. Campana, A.P. Beardmore, A.A. Breeveld, M.M. Chester, R. Corbet, S. Covino, J.R. Cummings, P. D'Avanzo, V. D'Elia, P. Esposito, P.A. Evans, D. Fugazza, J.M. Gelbord, K. Hiroi, S.T. Holland, K.Y. Huang, M. Im, G. Israel, Y. Jeon, Y.-B. Jeon, H.D. Jun, N. Kawai, J.H. Kim, H.A. Krimm, F.E. Marshall, P. Mészáros, H. Negoro, N. Omodei, W.-K. Park, J.S. Perkins, M. Sugizaki, H.-I. Sung, G. Tagliaferri, E. Troja, Y. Ueda, Y. Urata, R. Usui, L.A. Antonelli, S.D. Barthelmy, G. Cusumano, P. Giommi, A. Melandri, M. Perri, J.L. Racusin, B. Sbarufatti, M.H. Siegel, N. Gehrels, Relativistic jet activity from the tidal disruption of a star by a massive black hole. Nature 476, 421-424 (2011). doi:10.1038/nature10374

M. Cadolle Bel, P. Sizun, A. Goldwurm, J. Rodriguez, P. Laurent, A.A. Zdziarski, L. Foschini, P. Goldoni, C. Gouiffès, J. Malzac, E. Jourdain, J.-P. Roques, The broad-band spectrum of Cygnus X-1 measured by INTEGRAL. Astron. Astrophys. 446, 591-602 (2006). doi:10.1051/0004-6361:20053068

M. Cadolle Bel, J. Rodriguez, P. D'Avanzo, D.M. Russell, J. Tomsick, S. Corbel, F.W. Lewis, F. Rahoui, M. Buxton, P. Goldoni, E. Kuulkers, Overview of an extensive multiwavelength study of GX 339-4 during the 2010 outburst. Astron. Astrophys. 534, 119 (2011). doi:10.1051/0004-6361/201117684

P. Casella, A. Pe'er, On the Role of the Magnetic Field on Jet Emission in X-Ray Binaries. Astrophys. J. 703, 63-66 (2009). doi:10.1088/0004-637X/703/1/L63 
P. Casella, T.J. Maccarone, K. O'Brien, R.P. Fender, D.M. Russell, M. van der Klis, A. Pe'Er, D. Maitra, D. Altamirano, T. Belloni, G. Kanbach, M. Klein-Wolt, E. Mason, P. Soleri, A. Stefanescu, K. Wiersema, R. Wijnands, Fast infrared variability from a relativistic jet in GX 339-4. Mon. Not. R. Astron. Soc. 404, 21-25 (2010). doi:10.1111/j.17453933.2010.00826.x

A. Celotti, A.C. Fabian, The Kinetic Power and Luminosity of Parsecscale Radio Jets - an Argument for Heavy Jets. Mon. Not. R. Astron. Soc. 264, 228 (1993)

A. Celotti, G. Ghisellini, The power of blazar jets. Mon. Not. R. Astron. Soc. 385, 283-300 (2008). doi:10.1111/j.1365-2966.2007.12758.x

A. Celotti, G. Ghisellini, M. Chiaberge, Large-scale jets in active galactic nuclei: multiwavelength mapping. Mon. Not. R. Astron. Soc. 321, 1-5 (2001). doi:10.1046/j.13658711.2001.04160.x

M. Cerruti, C.D. Dermer, B. Lott, C. Boisson, A. Zech, Gamma-ray blazars near equipartition and the origin of the GeV spectral break in 3C 454.3. ArXiv e-prints (2013)

H.R. Christiansen, M. Orellana, G.E. Romero, High-energy neutrino emission from x-ray binaries. Physical Review D 73(6), 063012 (2006). doi:10.1103/PhysRevD.73.063012

D.F. Cioff, T.W. Jones, Internal Faraday rotation effects in transparent synchrotron sources. Astronomical J. 85, 368-375 (1980). doi:10.1086/112685

S. Corbel, R.P. Fender, A.K. Tzioumis, M. Nowak, V. McIntyre, P. Durouchoux, R. Sood, Coupling of the X-ray and radio emission in the black hole candidate and compact jet source GX 339-4. Astron. Astrophys. 359, 251-268 (2000)

S. Corbel, R.P. Fender, A.K. Tzioumis, J.A. Tomsick, J.A. Orosz, J.M. Miller, R. Wijnands, P. Kaaret, Large-Scale, Decelerating, Relativistic X-ray Jets from the Microquasar XTE J1550-564. Science 298, 196-199 (2002). doi:10.1126/science.1075857

S. Corbel, M.A. Nowak, R.P. Fender, A.K. Tzioumis, S. Markoff, Radio/X-ray correlation in the low/hard state of GX 339-4. Astron. Astrophys. 400, 1007-1012 (2003). doi:10.1051/00046361:20030090

S. Corbel, H. Aussel, J.W. Broderick, P. Chanial, M. Coriat, A.J. Maury, M.M. Buxton, J.A. Tomsick, A.K. Tzioumis, S. Markoff, J. Rodriguez, C.D. Bailyn, C. Brocksopp, R.P. Fender, P.O. Petrucci, M. Cadolle-Bel, D. Calvelo, L. Harvey-Smith, Formation of the compact jets in the black hole GX 339-4. Mon. Not. R. Astron. Soc. 431, 107-111 (2013). doi:10.1093/mnrasl/slt018

M. Coriat, S. Corbel, M.M. Buxton, C.D. Bailyn, J.A. Tomsick, E. Körding, E. Kalemci, The infrared/X-ray correlation of GX 339-4: probing hard X-ray emission in accreting black holes. Mon. Not. R. Astron. Soc. 400, 123-133 (2009). doi:10.1111/j.13652966.2009.15461.x

M. Coriat, S. Corbel, L. Prat, J.C.A. Miller-Jones, D. Cseh, A.K. Tzioumis, C. Brocksopp, J. Rodriguez, R.P. Fender, G.R. Sivakoff, Radiatively efficient accreting black holes in the hard state: the case study of H1743-322. Mon. Not. R. Astron. Soc. 414, 677-690 (2011). doi:10.1111/j.1365-2966.2011.18433.x

J.H. Croston, M.J. Hardcastle, D.E. Harris, E. Belsole, M. Birkinshaw, D.M. Worrall, An XRay Study of Magnetic Field Strengths and Particle Content in the Lobes of FR II Radio Sources. Astrophys. J. 626, 733-747 (2005). doi:10.1086/430170

F. Daigne, R. Mochkovitch, Gamma-ray bursts from internal shocks in a relativistic wind: temporal and spectral properties. Mon. Not. R. Astron. Soc. 296, 275-286 (1998). doi:10.1046/j.1365-8711.1998.01305.x

M. Del Santo, J. Malzac, R. Belmont, L. Bouchet, G. De Cesare, The magnetic field in the X-ray corona of Cygnus X-1. Mon. Not. R. Astron. Soc. 430, 209-220 (2013). doi:10.1093/mnras/sts574

C.D. Dermer, M. Böttcher, Gamma Rays from Compton Scattering in the Jets of Microquasars: Application to LS 5039. Astrophys. J. 643, 1081-1097 (2006). doi:10.1086/502966

C.D. Dermer, R. Schlickeiser, Model for the High-Energy Emission from Blazars. Astrophys. J. 416, 458 (1993). doi:10.1086/173251

C.D. Dermer, J.A. Miller, H. Li, Stochastic Particle Acceleration near Accreting Black Holes. Astrophys. J. 456, 106 (1996). doi:10.1086/176631

C.D. Dermer, R. Schlickeiser, A. Mastichiadis, High-energy gamma radiation from extragalactic radio sources. Astron. Astrophys. 256, 27-30 (1992)

C.D. Dermer, S.J. Sturner, R. Schlickeiser, Nonthermal Compton and Synchrotron Processes in the Jets of Active Galactic Nuclei. Astrophys. J. 109, 103 (1997). doi:10.1086/312972

D. Donato, G. Ghisellini, G. Tagliaferri, G. Fossati, Hard X-ray properties of blazars. 
Astron. Astrophys. 375, 739-751 (2001). doi:10.1051/0004-6361:20010675

R. Droulans, R. Belmont, J. Malzac, E. Jourdain, Variability and Spectral Modeling of the Hard X-ray Emission of GX 339-4 in a Bright Low/Hard State. Astrophys. J. 717, 10221036 (2010). doi:10.1088/0004-637X/717/2/1022

D.C. Ellison, G.P. Double, Diffusive shock acceleration in unmodified relativistic, oblique shocks. Astroparticle Physics 22, 323-338 (2004). doi:10.1016/j.astropartphys.2004.08.005

D.C. Ellison, S.P. Reynolds, F.C. Jones, First-order Fermi particle acceleration by relativistic shocks. Astrophys. J. 360, 702-714 (1990). doi:10.1086/169156

A.A. Esin, J.E. McClintock, R. Narayan, Advection-dominated Accretion and the Spectral States of Black Hole X-Ray Binaries: Application to Nova MUSCAE 1991. Astrophys. J. 489, 865-889 (1997). doi:10.1086/304829

A.A. Esin, J.E. McClintock, J.J. Drake, M.R. Garcia, C.A. Haswell, R.I. Hynes, M.P. Muno, Modeling the Low-State Spectrum of the X-Ray Nova XTE J1118+480. Astrophys. J. 555, 483-488 (2001). doi:10.1086/321450

H. Falcke, P.L. Biermann, The jet-disk symbiosis. I. Radio to X-ray emission models for quasars. Astron. Astrophys. 293, 665-682 (1995)

H. Falcke, P.L. Biermann, The jet/disk symbiosis. III. What the radio cores in GRS $1915+105$, NGC 4258, M 81 and SGR A* tell us about accreting black holes. Astron. Astrophys. 342, 49-56 (1999)

E.D. Feigelson, S.A. Laurent-Muehleisen, R.I. Kollgaard, E.B. Fomalont, Discovery of InverseCompton X-Rays in Radio Lobes. Astrophys. J. 449, 149 (1995). doi:10.1086/309642

R. Fender, Jets from X-ray Binaries, ed. by W.H.G. Lewin, M. van der Klis 2006, pp. 381-419

R. Fender, 'Disc-Jet' Coupling in Black Hole X-ray Binaries and Active Galactic Nuclei, in Lecture Notes in Physics, Berlin Springer Verlag, ed. by T. Belloni Lecture Notes in Physics, Berlin Springer Verlag, vol. 794, 2010, p. 115

R.P. Fender, Powerful jets from black hole X-ray binaries in low/hard X-ray states. Mon. Not. R. Astron. Soc. 322, 31-42 (2001). doi:10.1046/j.1365-8711.2001.04080.x

R.P. Fender, S.T. Garrington, D.J. McKay, T.W.B. Muxlow, G.G. Pooley, R.E. Spencer, A.M. Stirling, E.B. Waltman, MERLIN observations of relativistic ejections from GRS 1915+105. Mon. Not. R. Astron. Soc. 304, 865-876 (1999a). doi:10.1046/j.13658711.1999.02364.x

R. Fender, S. Corbel, T. Tzioumis, V. McIntyre, D. Campbell-Wilson, M. Nowak, R. Sood, R. Hunstead, A. Harmon, P. Durouchoux, W. Heindl, Quenching of the Radio Jet during the X-Ray High State of GX 339-4. Astrophys. J. 519, 165-168 (1999b). doi:10.1086/312128

E. Fermi, On the Origin of the Cosmic Radiation. Physical Review 75, 1169-1174 (1949). doi:10.1103/PhysRev.75.1169

E. Fermi, Galactic Magnetic Fields and the Origin of Cosmic Radiation. Astrophys. J. 119, 1 (1954). doi:10.1086/145789

J. Ferreira, C. Dougados, S. Cabrit, Which jet launching mechanism(s) in T Tauri stars? Astron. Astrophys. 453, 785-796 (2006). doi:10.1051/0004-6361:20054231

J.D. Finke, C.D. Dermer, M. Böttcher, Synchrotron Self-Compton Analysis of TeV X-RaySelected BL Lacertae Objects. Astrophys. J. 686, 181-194 (2008). doi:10.1086/590900

J.D. Finke, S. Razzaque, C.D. Dermer, Modeling the Extragalactic Background Light from Stars and Dust. Astrophys. J. 712, 238-249 (2010). doi:10.1088/0004-637X/712/1/238

G. Fossati, L. Maraschi, A. Celotti, A. Comastri, G. Ghisellini, A unifying view of the spectral energy distributions of blazars. Mon. Not. R. Astron. Soc. 299, 433-448 (1998). doi:10.1046/j.1365-8711.1998.01828.x

P.C. Fragile, D.L. Meier, General Relativistic Magnetohydrodynamic Simulations of the Hard State as a Magnetically Dominated Accretion Flow. Astrophys. J. 693, 771-783 (2009). doi:10.1088/0004-637X/693/1/771

J.T. Frederiksen, C.B. Hededal, T. Haugbølle, A. Nordlund, Magnetic Field Generation in Collisionless Shocks: Pattern Growth and Transport. Astrophys. J. 608, 13-16 (2004). doi:10.1086/421262

F. Frontera, A.A. Zdziarski, L. Amati, J. Mikołajewska, T. Belloni, S. Del Sordo, F. Haardt, E. Kuulkers, N. Masetti, M. Orlandini, E. Palazzi, A.N. Parmar, R.A. Remillard, A. Santangelo, L. Stella, A Measurement of the Broadband Spectrum of XTE J1118+480 with BeppoSAX and Its Astrophysical Implications. Astrophys. J. 561, 1006-1015 (2001). doi:10.1086/323258

T.K. Gaisser, F. Halzen, T. Stanev, Particle astrophysics with high energy neutrinos. Phys. Rep. 258, 173-236 (1995). doi:10.1016/0370-1573(95)00003-Y 
E. Gallo, Radio Emission and Jets from Microquasars, in Lecture Notes in Physics, Berlin Springer Verlag, ed. by T. Belloni Lecture Notes in Physics, Berlin Springer Verlag, vol. 794, 2010, p. 85

E. Gallo, R.P. Fender, G.G. Pooley, A universal radio-X-ray correlation in low/hard state black hole binaries. Mon. Not. R. Astron. Soc. 344, 60-72 (2003). doi:10.1046/j.13658711.2003.06791.x

E. Gallo, B.P. Miller, R. Fender, Assessing luminosity correlations via cluster analysis: evidence for dual tracks in the radio/X-ray domain of black hole X-ray binaries. Mon. Not. R. Astron. Soc. 423, 590-599 (2012). doi:10.1111/j.1365-2966.2012.20899.x

E. Gallo, S. Migliari, S. Markoff, J.A. Tomsick, C.D. Bailyn, S. Berta, R. Fender, J.C.A. Miller-Jones, The Spectral Energy Distribution of Quiescent Black Hole X-Ray Binaries: New Constraints from Spitzer. Astrophys. J. 670, 600-609 (2007). doi:10.1086/521524

P. Gandhi, V.S. Dhillon, M. Durant, A.C. Fabian, A. Kubota, K. Makishima, J. Malzac, T.R. Marsh, J.M. Miller, T. Shahbaz, H.C. Spruit, P. Casella, Rapid optical and X-ray timing observations of GX339-4: multicomponent optical variability in the low/hard state. Mon. Not. R. Astron. Soc. 407, 2166-2192 (2010). doi:10.1111/j.1365-2966.2010.17083.x

P. Gandhi, A.W. Blain, D.M. Russell, P. Casella, J. Malzac, S. Corbel, P. D'Avanzo, F.W. Lewis, S. Markoff, M. Cadolle Bel, P. Goldoni, S. Wachter, D. Khangulyan, A. Mainzer, A Variable Mid-infrared Synchrotron Break Associated with the Compact Jet in GX 339-4. Astrophys. J. 740, 13 (2011). doi:10.1088/2041-8205/740/1/L13

M. Georganopoulos, D. Kazanas, Decelerating Flows in TeV Blazars: A Resolution to the BL Lacertae-FR I Unification Problem. Astrophys. J. 594, 27-30 (2003). doi:10.1086/378557

M. Georganopoulos, R.M. Sambruna, D. Kazanas, A.N. Cillis, C.C. Cheung, E.S. Perlman, K.M. Blundell, D.S. Davis, A Novel Method for Measuring the Extragalactic Background Light: Fermi Application to the Lobes of Fornax A. Astrophys. J. 686, 5-8 (2008). doi: $10.1086 / 592833$

G. Ghisellini, Jetted Active Galactic Nuclei. International Journal of Modern Physics Conference Series 8, 1 (2012). doi:10.1142/S2010194512004345

G. Ghisellini, P. Madau, On the origin of the gamma-ray emission in blazars. Mon. Not. R. Astron. Soc. 280, 67-76 (1996)

G. Ghisellini, F. Tavecchio, G. Ghirlanda, Jet and accretion power in the most powerful Fermi blazars. Mon. Not. R. Astron. Soc. 399, 2041-2054 (2009). doi:10.1111/j.13652966.2009.15397.x

G. Ghisellini, F. Tavecchio, L. Foschini, G. Ghirlanda, L. Maraschi, A. Celotti, General physical properties of bright Fermi blazars. Mon. Not. R. Astron. Soc. 402, 497-518 (2010). doi:10.1111/j.1365-2966.2009.15898.x

D. Giannios, Spectra of black-hole binaries in the low/hard state: From radio to X-rays. Astron. Astrophys. 437, 1007-1015 (2005). doi:10.1051/0004-6361:20041491

D. Giannios, B.D. Metzger, Radio transients from stellar tidal disruption by massive black holes. Mon. Not. R. Astron. Soc. 416, 2102-2107 (2011). doi:10.1111/j.13652966.2011.19188.x

D. Giannios, D.A. Uzdensky, M.C. Begelman, Fast TeV variability in blazars: jets in a jet. Mon. Not. R. Astron. Soc. 395, 29-33 (2009). doi:10.1111/j.1745-3933.2009.00635.x

V.L. Ginzburg, S.I. Syrovatskii, Cosmic Magnetobremsstrahlung (synchrotron Radiation). Annu. Rev. Astron. Astrophys. 3, 297 (1965). doi:10.1146/annurev.aa.03.090165.001501

S. Gupta, M. Böttcher, C.D. Dermer, Time-dependent Synchrotron and Compton Spectra from Jets of Microquasars. Astrophys. J. 644, 409-423 (2006). doi:10.1086/503552

F. Haardt, L. Maraschi, X-ray spectra from two-phase accretion disks. Astrophys. J. 413, 507-517 (1993). doi:10.1086/173020

D.C. Hannikainen, R.W. Hunstead, D. Campbell-Wilson, R.K. Sood, MOST radio monitoring of GX 339-4. Astron. Astrophys. 337, 460-464 (1998)

M.J. Hardcastle, C.C. Cheung, I.J. Feain, L. Stawarz, High-energy particle acceleration and production of ultra-high-energy cosmic rays in the giant lobes of Centaurus A. Mon. Not. R. Astron. Soc. 393, 1041-1053 (2009). doi:10.1111/j.1365-2966.2008.14265.x

D.E. Harris, H. Krawczynski, X-Ray Emission from Extragalactic Jets. Annu. Rev. Astron. Astrophys. 44, 463-506 (2006). doi:10.1146/annurev.astro.44.051905.092446

R.C. Hartman, M. Böttcher, G. Aldering, H. Aller, M. Aller, D.E. Backman, T.J. Balonek, D.L. Bertsch, et. al., Multiepoch Multiwavelength Spectra and Models for Blazar 3C 279. Astrophys. J. 553, 683-694 (2001). doi:10.1086/320970 
T. Haugbølle, Three-dimensional Modeling of Relativistic Collisionless Ion-electron Shocks. Astrophys. J. 739, 42 (2011). doi:10.1088/2041-8205/739/2/L42

S. Heinz, R.A. Sunyaev, The non-linear dependence of flux on black hole mass and accretion rate in core-dominated jets. Mon. Not. R. Astron. Soc. 343, 59-64 (2003). doi:10.1046/j.1365-8711.2003.06918.x

R.M. Hjellming, K.J. Johnston, Radio emission from conical jets associated with X-ray binaries. Astrophys. J. 328, 600-609 (1988). doi:10.1086/166318

J. Homan, M. Buxton, S. Markoff, C.D. Bailyn, E. Nespoli, T. Belloni, Multiwavelength Observations of the 2002 Outburst of GX 339-4: Two Patterns of X-Ray-Optical/Near-Infrared Behavior. Astrophys. J. 624, 295-306 (2005). doi:10.1086/428722

R.I. Hynes, C.W. Mauche, C.A. Haswell, C.R. Shrader, W. Cui, S. Chaty, The X-Ray Transient XTE J1118+480: Multiwavelength Observations of a Low-State Minioutburst. Astrophys. J. 539, 37-40 (2000). doi:10.1086/312836

A. Ingram, C. Done, A physical model for the continuum variability and quasi-periodic oscillation in accreting black holes. Mon. Not. R. Astron. Soc. 415, 2323-2335 (2011). doi:10.1111/j.1365-2966.2011.18860.x

A. Ingram, C. Done, Modelling variability in black hole binaries: linking simulations to observations. Mon. Not. R. Astron. Soc. 419, 2369-2378 (2012). doi:10.1111/j.13652966.2011.19885.x

O. Jamil, R.P. Fender, C.R. Kaiser, iShocks: X-ray binary jets with an internal shocks model. Mon. Not. R. Astron. Soc. 401, 394-404 (2010). doi:10.1111/j.1365-2966.2009.15652.x

A. Joinet, E. Kalemci, F. Senziani, Hard X-Ray Emission of the Microquasar GRO J1655-40 during the Rise of Its 2005 Outburst. Astrophys. J. 679, 655-663 (2008). doi:10.1086/533512

F.C. Jones, D.C. Ellison, The plasma physics of shock acceleration. Space Science Rev. 58, 259-346 (1991). doi:10.1007/BF01206003

T.W. Jones, P.E. Hardee, Maxwellian synchrotron sources. Astrophys. J. 228, 268-278 (1979). doi:10.1086/156843

E. Jourdain, J.P. Roques, M. Chauvin, D.J. Clark, Separation of Two Contributions to the High Energy Emission of Cygnus X-1: Polarization Measurements with INTEGRAL SPI. Astrophys. J. 761, 27 (2012). doi:10.1088/0004-637X/761/1/27

C.R. Kaiser, The flat synchrotron spectra of partially self-absorbed jets revisited. Mon. Not. R. Astron. Soc. 367, 1083-1094 (2006). doi:10.1111/j.1365-2966.2006.10030.x

C.R. Kaiser, R. Sunyaev, H.C. Spruit, Internal shock model for microquasars. Astron. Astrophys. 356, 975-988 (2000)

G. Kanbach, C. Straubmeier, H.C. Spruit, T. Belloni, Correlated fast X-ray and optical variability in the black-hole candidate XTE J1118+480. Nature 414, 180-182 (2001)

J. Kataoka, Ł. Stawarz, X-Ray Emission Properties of Large-Scale Jets, Hot Spots, and Lobes in Active Galactic Nuclei. Astrophys. J. 622, 797-810 (2005). doi:10.1086/428083

U. Keshet, B. Katz, A. Spitkovsky, E. Waxman, Magnetic Field Evolution in Relativistic Unmagnetized Collisionless Shocks. Astrophys. J. 693, 127-130 (2009). doi:10.1088/0004$637 \mathrm{X} / 693 / 2 / \mathrm{L} 127$

M. Kino, F. Takahara, M. Kusunose, Energetics of TeV Blazars and Physical Constraints on Their Emission Regions. Astrophys. J. 564, 97-107 (2002). doi:10.1086/323363

J.G. Kirk, F.M. Rieger, A. Mastichiadis, Particle acceleration and synchrotron emission in blazar jets. Astron. Astrophys. 333, 452-458 (1998)

J.G. Kirk, A.W. Guthmann, Y.A. Gallant, A. Achterberg, Particle Acceleration at Ultrarelativistic Shocks: An Eigenfunction Method. Astrophys. J. 542, 235-242 (2000). doi:10.1086/309533

D.A. Kniffen, D.L. Bertsch, C.E. Fichtel, R.C. Hartman, S.D. Hunter, G. Kanbach, P.W. Kwok, Y.C. Lin, J.R. Mattox, H.A. Mayer-Hasselwander, P.F. Michelson, C. von Montigny, P.L. Nolan, K. Pinkau, E. Schneid, P. Sreekumar, D.J. Thompson, Time variability in the gamma-ray emission of 3C 279. Astrophys. J. 411, 133-136 (1993). doi:10.1086/172813

H.B.J. Koers, A. Pe'er, R.A.M.J. Wijers, Parameterization of the energy and rapidity distributions of secondary pions and kaons produced in energetic proton-proton collisions. ArXiv High Energy Physics - Phenomenology e-prints (2006)

S.S. Komissarov, M.V. Barkov, N. Vlahakis, A. Königl, Magnetic acceleration of relativistic active galactic nucleus jets. Mon. Not. R. Astron. Soc. 380, 51-70 (2007). doi:10.1111/j.1365-2966.2007.12050.x

A. Konigl, Relativistic jets as X-ray and gamma-ray sources. Astrophys. J. 243, 700-709 
(1981). doi:10.1086/158638

K. Kotera, A.V. Olinto, The Astrophysics of Ultrahigh-Energy Cosmic Rays. Annu. Rev. Astron. Astrophys. 49, 119-153 (2011). doi:10.1146/annurev-astro-081710102620

O. Kotov, E. Churazov, M. Gilfanov, On the X-ray time-lags in the black hole candidates. Mon. Not. R. Astron. Soc. 327, 799-807 (2001). doi:10.1046/j.1365-8711.2001.04769.x

H. Krawczynski, P.S. Coppi, F. Aharonian, Time-dependent modelling of the Markarian 501 X-ray and $\mathrm{TeV}$ gamma-ray data taken during 1997 March and April. Mon. Not. R. Astron. Soc. 336, 721-735 (2002). doi:10.1046/j.1365-8711.2002.05750.x

P. Laurent, J. Rodriguez, J. Wilms, M. Cadolle Bel, K. Pottschmidt, V. Grinberg, Polarized Gamma-Ray Emission from the Galactic Black Hole Cygnus X-1. Science 332, 438 (2011). doi:10.1126/science. 1200848

A. Lazarian, G. Kowal, E. Vishniac, E. de Gouveia Dal Pino, Fast magnetic reconnection and energetic particle acceleration. Planetary and Space Science 59, 537-546 (2011). doi:10.1016/j.pss.2010.07.020

A.J. Levan, N.R. Tanvir, S.B. Cenko, D.A. Perley, K. Wiersema, J.S. Bloom, A.S. Fruchter, A.d.U. Postigo, P.T. O'Brien, N. Butler, A.J. van der Horst, G. Leloudas, A.N. Morgan, K. Misra, G.C. Bower, J. Farihi, R.L. Tunnicliffe, M. Modjaz, J.M. Silverman, J. Hjorth, C. Thöne, A. Cucchiara, J.M.C. Cerón, A.J. Castro-Tirado, J.A. Arnold, M. Bremer, J.P. Brodie, T. Carroll, M.C. Cooper, P.A. Curran, R.M. Cutri, J. Ehle, D. Forbes, J. Fynbo, J. Gorosabel, J. Graham, D.I. Hoffman, S. Guziy, P. Jakobsson, A. Kamble, T. Kerr, M.M. Kasliwal, C. Kouveliotou, D. Kocevski, N.M. Law, P.E. Nugent, E.O. Ofek, D. Poznanski, R.M. Quimby, E. Rol, A.J. Romanowsky, R. Sánchez-Ramírez, S. Schulze, N. Singh, L. van Spaandonk, R.L.C. Starling, R.G. Strom, J.C. Tello, O. Vaduvescu, P.J. Wheatley, R.A.M.J. Wijers, J.M. Winters, D. Xu, An Extremely Luminous Panchromatic Outburst from the Nucleus of a Distant Galaxy. Science 333, 199 (2011). doi:10.1126/science. 1207143

A. Levinson, High-Energy Aspects of Astrophysical Jets. International Journal of Modern Physics A 21, 6015-6054 (2006). doi:10.1142/S0217751X06035063

A. Levinson, R. Blandford, On the Jets Associated with Galactic Superluminal Sources. Astrophys. J. 456, 29 (1996). doi:10.1086/309851

A. Levinson, D. Eichler, Baryon Purity in Cosmological Gamma-Ray Bursts as a Manifestation of Event Horizons. Astrophys. J. 418, 386 (1993). doi:10.1086/173397

A. Levinson, E. Waxman, Probing Microquasars with TeV Neutrinos. Physical Review Letters 87(17), 171101 (2001). doi:10.1103/PhysRevLett.87.171101

D. Liu, A. Pe'er, A. Loeb, A two-component jet model for the tidal disruption event Swift J164449.3+573451. ArXiv e-prints (2012)

M. Livio, G.I. Ogilvie, J.E. Pringle, Extracting Energy from Black Holes: The Relative Importance of the Blandford-Znajek Mechanism. Astrophys. J. 512, 100-104 (1999). doi:10.1086/306777

M.S. Longair, High Energy Astrophysics 2011

R.V.E. Lovelace, Dynamo model of double radio sources. Nature 262, 649-652 (1976). doi: $10.1038 / 262649 \mathrm{a} 0$

Y. Lyubarsky, M. Liverts, Particle Acceleration in the Driven Relativistic Reconnection. Astrophys. J. 682, 1436-1442 (2008). doi:10.1086/589640

M. Lyutikov, Role of reconnection in AGN jets. New Astronomy Rev. 47, 513-515 (2003). doi:10.1016/S1387-6473(03)00083-6

T.J. Maccarone, Constraints on jet X-ray emission in low/hard-state X-ray binaries. Mon. Not. R. Astron. Soc. 360, 68-72 (2005). doi:10.1111/j.1745-3933.2005.00047.x

T.J. Maccarone, Jets from Galactic binaries. ArXiv e-prints (2012)

P. Magdziarz, A.A. Zdziarski, Angle-dependent Compton reflection of X-rays and gamma-rays. Mon. Not. R. Astron. Soc. 273, 837-848 (1995)

D. Maitra, S. Markoff, C. Brocksopp, M. Noble, M. Nowak, J. Wilms, Constraining jet/disc geometry and radiative processes in stellar black holes XTE J1118+480 and GX 339-4. Mon. Not. R. Astron. Soc. 398, 1638-1650 (2009). doi:10.1111/j.1365-2966.2009.14896.x

J. Malzac, Internal shocks at the origin of the flat spectral energy distribution of compact jets. Mon. Not. R. Astron. Soc. 429, 20-24 (2013). doi:10.1093/mnrasl/sls017

J. Malzac, R. Belmont, The synchrotron boiler and the spectral states of black hole binaries. Mon. Not. R. Astron. Soc. 392, 570-589 (2009). doi:10.1111/j.1365-2966.2008.14142.x

J. Malzac, R. Belmont, A.C. Fabian, Energetics of a black hole: constraints on the jet velocity 
and the nature of the X-ray emitting region in Cyg X-1. Mon. Not. R. Astron. Soc. 400, 1512-1520 (2009). doi:10.1111/j.1365-2966.2009.15553.x

K. Mannheim, The proton blazar. Astron. Astrophys. 269, 67-76 (1993)

K. Mannheim, P.L. Biermann, Gamma-ray flaring of 3C 279 - A proton-initiated cascade in the jet? Astron. Astrophys. 253, 21-24 (1992)

L. Maraschi, G. Ghisellini, A. Celotti, A jet model for the gamma-ray emitting blazar 3C 279. Astrophys. J. 397, 5-9 (1992). doi:10.1086/186531

S. Markoff, From Multiwavelength to Mass Scaling: Accretion and Ejection in Microquasars and Agn, in Lecture Notes in Physics, Berlin Springer Verlag, ed. by T. Belloni Lecture Notes in Physics, Berlin Springer Verlag, vol. 794, 2010, p. 143

S. Markoff, H. Falcke, R. Fender, A jet model for the broadband spectrum of XTE $\mathrm{J} 1118+480$. Synchrotron emission from radio to X-rays in the Low/Hard spectral state. Astron. Astrophys. 372, 25-28 (2001). doi:10.1051/0004-6361:20010420

S. Markoff, M.A. Nowak, J. Wilms, Going with the Flow: Can the Base of Jets Subsume the Role of Compact Accretion Disk Coronae? Astrophys. J. 635, 1203-1216 (2005). doi:10.1086/497628

S. Markoff, M. Nowak, S. Corbel, R. Fender, H. Falcke, Exploring the role of jets in the radio/Xray correlations of GX 339-4. Astron. Astrophys. 397, 645-658 (2003). doi:10.1051/00046361:20021497

A.P. Marscher, Relativistic jets and the continuum emission in QSOs. Astrophys. J. 235, 386391 (1980). doi:10.1086/157642

A.P. Marscher, Jets in Active Galactic Nuclei. ArXiv e-prints (2009)

A.P. Marscher, W.K. Gear, Models for high-frequency radio outbursts in extragalactic sources, with application to the early 1983 millimeter-to-infrared flare of 3C 273. Astrophys. J. 298, 114-127 (1985). doi:10.1086/163592

J.E. McClintock, C.A. Haswell, M.R. Garcia, J.J. Drake, R.I. Hynes, H.L. Marshall, M.P. Muno, S. Chaty, P.M. Garnavich, P.J. Groot, W.H.G. Lewin, C.W. Mauche, J.M. Miller, G.G. Pooley, C.R. Shrader, S.D. Vrtilek, Complete and Simultaneous Spectral Observations of the Black Hole X-Ray Nova XTE J1118+480. Astrophys. J. 555, 477-482 (2001). doi:10.1086/321449

J.C. McKinney, Total and Jet Blandford-Znajek Power in the Presence of an Accretion Disk. Astrophys. J. 630, 5-8 (2005). doi:10.1086/468184

J.C. McKinney, General relativistic magnetohydrodynamic simulations of the jet formation and large-scale propagation from black hole accretion systems. Mon. Not. R. Astron. Soc. 368, 1561-1582 (2006). doi:10.1111/j.1365-2966.2006.10256.x

J.C. McKinney, C.F. Gammie, A Measurement of the Electromagnetic Luminosity of a Kerr Black Hole. Astrophys. J. 611, 977-995 (2004). doi:10.1086/422244

J.C. McKinney, D.A. Uzdensky, A reconnection switch to trigger gamma-ray burst jet dissipation. Mon. Not. R. Astron. Soc. 419, 573-607 (2012). doi:10.1111/j.13652966.2011.19721.x

M.V. Medvedev, A. Loeb, Generation of Magnetic Fields in the Relativistic Shock of GammaRay Burst Sources. Astrophys. J. 526, 697-706 (1999). doi:10.1086/308038

D.L. Meier, The Association of Jet Production with Geometrically Thick Accretion Flows and Black Hole Rotation. Astrophys. J. 548, 9-12 (2001). doi:10.1086/318921

D.L. Meier, S. Koide, Y. Uchida, Magnetohydrodynamic Production of Relativistic Jets. Science 291, 84-92 (2001). doi:10.1126/science.291.5501.84

P. Mészáros, Gamma-ray bursts. Reports on Progress in Physics 69, 2259-2321 (2006). doi:10.1088/0034-4885/69/8/R01

P. Meszaros, M.J. Rees, Optical and Long-Wavelength Afterglow from Gamma-Ray Bursts. Astrophys. J. 476, 232 (1997). doi:10.1086/303625

S. Migliari, J.A. Tomsick, S. Markoff, E. Kalemci, C.D. Bailyn, M. Buxton, S. Corbel, R.P. Fender, P. Kaaret, Tracing the Jet Contribution to the Mid-IR over the 2005 Outburst of GRO J1655-40 via Broadband Spectral Modeling. Astrophys. J. 670, 610-623 (2007). doi:10.1086/522023

J.C.A. Miller-Jones, D.G. McCormick, R.P. Fender, R.E. Spencer, T.W.B. Muxlow, G.G. Pooley, Multiple relativistic outbursts of GRS1915+105: radio emission and internal shocks. Mon. Not. R. Astron. Soc. 363, 867-881 (2005). doi:10.1111/j.1365-2966.2005.09488.x

J.C.A. Miller-Jones, G.R. Sivakoff, D. Altamirano, M. Coriat, S. Corbel, V. Dhawan, H.A. Krimm, R.A. Remillard, M.P. Rupen, D.M. Russell, R.P. Fender, S. Heinz, E.G. Körding, D. Maitra, S. Markoff, S. Migliari, C.L. Sarazin, V. Tudose, Disc-jet coupling in the 2009 
outburst of the black hole candidate H1743-322. Mon. Not. R. Astron. Soc. 421, 468-485 (2012). doi:10.1111/j.1365-2966.2011.20326.x

I.F. Mirabel, L.F. Rodríguez, A superluminal source in the Galaxy. Nature 371, 46-48 (1994). doi: $10.1038 / 371046 \mathrm{a} 0$

A. Mücke, R.J. Protheroe, R. Engel, J.P. Rachen, T. Stanev, BL Lac objects in the synchrotron proton blazar model. Astroparticle Physics 18, 593-613 (2003). doi:10.1016/S09276505(02)00185-8

K. Murase, C.D. Dermer, H. Takami, G. Migliori, Blazars as Ultra-high-energy Cosmic-ray Sources: Implications for TeV Gamma-Ray Observations. Astrophys. J. 749, 63 (2012). doi:10.1088/0004-637X/749/1/63

R. Narayan, J.E. McClintock, Observational evidence for a correlation between jet power and black hole spin. Mon. Not. R. Astron. Soc. 419, 69-73 (2012). doi:10.1111/j.17453933.2011.01181.x

A.Y. Neronov, D.V. Semikoz, I.I. Tkachev, Ultra-high energy cosmic ray production in the polar cap regions of black hole magnetospheres. New Journal of Physics 11(6), 065015 (2009). doi:10.1088/1367-2630/11/6/065015

K.-I. Nishikawa, P. Hardee, G. Richardson, R. Preece, H. Sol, G.J. Fishman, Particle Acceleration in Relativistic Jets Due to Weibel Instability. Astrophys. J. 595, 555-563 (2003). doi: $10.1086 / 377260$

K.-I. Nishikawa, P. Hardee, G. Richardson, R. Preece, H. Sol, G.J. Fishman, Particle Acceleration and Magnetic Field Generation in Electron-Positron Relativistic Shocks. Astrophys. J. 622, 927-937 (2005). doi:10.1086/428394

C.A. Norman, D.B. Melrose, A. Achterberg, The Origin of Cosmic Rays above $1018.5 \mathrm{eV}$. Astrophys. J. 454, 60 (1995). doi:10.1086/176465

H. Oda, M. Machida, K.E. Nakamura, R. Matsumoto, Thermal Equilibria of Optically Thin, Magnetically Supported, Two-temperature, Black Hole Accretion Disks. Astrophys. J. 712, 639-652 (2010). doi:10.1088/0004-637X/712/1/639

S.P. O'Sullivan, D.C. Gabuzda, Magnetic field strength and spectral distribution of six parsec-scale active galactic nuclei jets. Mon. Not. R. Astron. Soc. 400, 26-42 (2009). doi:10.1111/j.1365-2966.2009.15428.x

N.I. Palma, M. Böttcher, I. de la Calle, I. Agudo, M. Aller, H. Aller, U. Bach, E. Benítez, C.S. Buemi, L. Escande, J.L. Gómez, M.A. Gurwell, J. Heidt, D. Hiriart, S.G. Jorstad, M. Joshi, A. Lähteenmäki, V.M. Larionov, P. Leto, Y. Li, J.M. López, B. Lott, G. Madejski, A.P. Marscher, D.A. Morozova, C.M. Raiteri, V. Roberts, M. Tornikoski, C. Trigilio, G. Umana, M. Villata, D. Wylezalek, Multiwavelength Observations of the Gamma-Ray Blazar PKS 0528+134 in Quiescence. Astrophys. J. 735, 60 (2011). doi:10.1088/0004-637X/735/1/60

A. Pe'er, P. Casella, A Model for Emission from Jets in X-Ray Binaries: Consequences of a Single Acceleration Episode. Astrophys. J. 699, 1919-1937 (2009). doi:10.1088/0004$637 \mathrm{X} / 699 / 2 / 1919$

A. Pe'er, A. Loeb, Constraining sources of ultra high energy cosmic rays using high energy observations with the Fermi satellite. J. Cosmol. Astropart. Phys. 3, 7 (2012). doi:10.1088/1475-7516/2012/03/007

A. Pe'er, S. Markoff, X-Ray Emission from Transient Jet Model in Black Hole Binaries. Astrophys. J. 753, 177 (2012). doi:10.1088/0004-637X/753/2/177

A. Pe'er, E. Waxman, Prompt Gamma-Ray Burst Spectra: Detailed Calculations and the Effect of Pair Production. Astrophys. J. 613, 448-459 (2004). doi:10.1086/422989

A. Pe'er, E. Waxman, High-Energy Photon Emission in the Early Afterglow of GRBs. Astrophys. J. 633, 1018-1026 (2005a). doi:10.1086/468175

A. Pe'er, E. Waxman, Time-dependent Numerical Model for the Emission of Radiation from Relativistic Plasma. Astrophys. J. 628, 857-866 (2005b). doi:10.1086/431139

P.O. Petrucci, J. Ferreira, G. Henri, J. Malzac, C. Foellmi, Relevance of jet emitting disc physics to microquasars: application to Cygnus X-1. Astron. Astrophys. 522, 38 (2010). doi:10.1051/0004-6361/201014753

T. Piran, The physics of gamma-ray bursts. Reviews of Modern Physics 76, 1143-1210 (2004). doi:10.1103/RevModPhys.76.1143

J. Poutanen, Accretion Disc-corona Models and X/ $\gamma$-ray Spectra of Accreting Black holes, in Theory of Black Hole Accretion Disks, ed. by M. A. Abramowicz, G. Bjornsson, \& J. E. Pringle, 1998, p. 100

J. Poutanen, I. Vurm, On the Origin of Spectral States in Accreting Black Holes. Astrophys. J. 690, 97-100 (2009). doi:10.1088/0004-637X/690/2/L97 
J. Poutanen, A.A. Zdziarski, Radiative Processes in Microquasars, in New Views on Microquasars, ed. by P. Durouchoux, Y. Fuchs, J. Rodriguez, 2003, p. 95

R.E. Pudritz, M.J. Hardcastle, D.C. Gabuzda, Magnetic Fields in Astrophysical Jets: From Launch to Termination. Space Science Rev. 169, 27-72 (2012). doi:10.1007/s11214-0129895-Z

E. Qiao, B.F. Liu, A Model for the Correlation of Hard X-Ray Index with Eddington Ratio in Black Hole X-Ray Binaries. Astrophys. J. 764, 2 (2013). doi:10.1088/0004-637X/764/1/2

J.P. Rachen, P. Mészáros, Photohadronic neutrinos from transients in astrophysical sources. Physical Review D 58(12), 123005 (1998). doi:10.1103/PhysRevD.58.123005

F. Rahoui, J.C. Lee, S. Heinz, D.C. Hines, K. Pottschmidt, J. Wilms, V. Grinberg, A Multiwavelength Study of Cygnus X-1: The First Mid-infrared Spectroscopic Detection of Compact Jets. Astrophys. J. 736, 63 (2011). doi:10.1088/0004-637X/736/1/63

F. Rahoui, M. Coriat, S. Corbel, M. Cadolle Bel, J.A. Tomsick, J.C. Lee, J. Rodriguez, D.M. Russell, S. Migliari, Optical and near-infrared spectroscopy of the black hole GX 339-4 - I. A focus on the continuum in the low/hard and high/soft states. Mon. Not. R. Astron. Soc. 422, 2202-2212 (2012). doi:10.1111/j.1365-2966.2012.20763.x

S. Rawlings, R. Saunders, Evidence for a common central-engine mechanism in all extragalactic radio sources. Nature 349, 138-140 (1991). doi:10.1038/349138a0

M.J. Rees, Appearance of Relativistically Expanding Radio Sources. Nature 211, 468-470 (1966). doi:10.1038/211468a0

M.J. Rees, P. Meszaros, Unsteady outflow models for cosmological gamma-ray bursts. Astrophys. J. 430, 93-96 (1994). doi:10.1086/187446

W. Reich, H. Steppe, R. Schlickeiser, P. Reich, M. Pohl, H.P. Reuter, G. Kanbach, V. Schonfelder, The Radio State of Extragalactic Gamma-Ray Sources Detected by CGRO. Astron. Astrophys. 273, 65 (1993)

R.C. Reis, J.M. Miller, A.C. Fabian, Thermal emission from the stellar-mass black hole binary XTE J1118+480 in the low/hard state. Mon. Not. R. Astron. Soc. 395, $52-56$ (2009). doi:10.1111/j.1745-3933.2009.00640.x

S.P. Reynolds, Theoretical studies of compact radio sources. I - Synchrotron radiation from relativistic flows. Astrophys. J. 256, 13-37 (1982). doi:10.1086/159881

L.F. Rodríguez, I.F. Mirabel, Repeated Relativistic Ejections in GRS 1915+105. Astrophys. J. 511, 398-404 (1999). doi:10.1086/306642

L.F. Rodriguez, E. Gerard, I.F. Mirabel, Y. Gomez, A. Velazquez, Radio Monitoring of GRS 1915+105. Astrophys. J. 101, 173 (1995). doi:10.1086/192236

M.M. Romanova, R.V.E. Lovelace, Magnetic field, reconnection, and particle acceleration in extragalactic jets. Astron. Astrophys. 262, 26-36 (1992)

G.E. Romero, H.R. Christiansen, M. Orellana, Hadronic High-Energy Gamma-Ray Emission from the Microquasar LS I +61 303. Astrophys. J. 632, 1093-1098 (2005). doi:10.1086/444446

D.M. Russell, E. Gallo, R.P. Fender, Observational constraints on the powering mechanism of transient relativistic jets. Mon. Not. R. Astron. Soc. 431, 405-414 (2013). doi:10.1093/mnras/stt176

D.M. Russell, R.P. Fender, R.I. Hynes, C. Brocksopp, J. Homan, P.G. Jonker, M.M. Buxton, Global optical/infrared-X-ray correlations in X-ray binaries: quantifying disc and jet contributions. Mon. Not. R. Astron. Soc. 371, 1334-1350 (2006). doi:10.1111/j.13652966.2006.10756.x

D.M. Russell, F. Lewis, P. Roche, J.S. Clark, E. Breedt, R.P. Fender, A long-term opticalX-ray correlation in 4U 1957+11. Mon. Not. R. Astron. Soc. 402, 2671-2681 (2010). doi:10.1111/j.1365-2966.2009.16098.x

D.M. Russell, T.D. Russell, J.C.A. Miller-Jones, K. O’Brien, R. Soria, G.R. Sivakoff, T. SlavenBlair, F. Lewis, S. Markoff, J. Homan, D. Altamirano, P.A. Curran, M.P. Rupen, T.M. Belloni, M. Cadolle Bel, P. Casella, S. Corbel, V. Dhawan, R.P. Fender, E. Gallo, P. Gandhi, S. Heinz, E.G. Körding, H.A. Krimm, D. Maitra, S. Migliari, R.A. Remillard, C.L. Sarazin, T. Shahbaz, V. Tudose, An Evolving Compact Jet in the Black Hole X-Ray Binary MAXI J1836-194. Astrophys. J. 768, 35 (2013). doi:10.1088/2041-8205/768/2/L35

G.B. Rybicki, A.P. Lightman, Radiative Processes in Astrophysics 1979

R.M. Sambruna, J.K. Gambill, L. Maraschi, F. Tavecchio, R. Cerutti, C.C. Cheung, C.M. Urry, G. Chartas, A Survey of Extended Radio Jets with Chandra and the Hubble Space Telescope. Astrophys. J. 608, 698-720 (2004). doi:10.1086/383124

R. Santana, R. Barniol Duran, P. Kumar, Magnetic Fields In Relativistic Collisionless Shocks, 
in American Astronomical Society Meeting Abstracts. American Astronomical Society Meeting Abstracts, vol. 221, 2013, pp. 438-01

R. Sari, A.A. Esin, On the Synchrotron Self-Compton Emission from Relativistic Shocks and Its Implications for Gamma-Ray Burst Afterglows. Astrophys. J. 548, 787-799 (2001). doi:10.1086/319003

R. Sari, T. Piran, R. Narayan, Spectra and Light Curves of Gamma-Ray Burst Afterglows. Astrophys. J. 497, 17 (1998). doi:10.1086/311269

M. Sikora, M.C. Begelman, M.J. Rees, Comptonization of diffuse ambient radiation by a relativistic jet: The source of gamma rays from blazars? Astrophys. J. 421, 153-162 (1994). doi:10.1086/173633

L.O. Silva, R.A. Fonseca, J.W. Tonge, J.M. Dawson, W.B. Mori, M.V. Medvedev, Interpenetrating Plasma Shells: Near-equipartition Magnetic Field Generation and Nonthermal Particle Acceleration. Astrophys. J. 596, 121-124 (2003). doi:10.1086/379156

L. Sironi, A. Spitkovsky, Particle Acceleration in Relativistic Magnetized Collisionless Pair Shocks: Dependence of Shock Acceleration on Magnetic Obliquity. Astrophys. J. 698, 1523-1549 (2009). doi:10.1088/0004-637X/698/2/1523

L. Sironi, A. Spitkovsky, Particle Acceleration in Relativistic Magnetized Collisionless Electron-Ion Shocks. Astrophys. J. 726, 75 (2011). doi:10.1088/0004-637X/726/2/75

M.A. Sobolewska, A. Siemiginowska, G. Migliori, Ł. Stawarz, M. Jamrozy, D. Evans, C.C. Cheung, Nuclear X-Ray Properties of the Peculiar Radio-loud Hidden AGN 4C+29.30. Astrophys. J. 758, 90 (2012). doi:10.1088/0004-637X/758/2/90

M. Spada, G. Ghisellini, D. Lazzati, A. Celotti, Internal shocks in the jets of radio-loud quasars. Mon. Not. R. Astron. Soc. 325, 1559-1570 (2001). doi:10.1046/j.1365-8711.2001.04557.x

A. Spitkovsky, On the Structure of Relativistic Collisionless Shocks in Electron-Ion Plasmas. Astrophys. J. 673, 39-42 (2008a). doi:10.1086/527374

A. Spitkovsky, Particle Acceleration in Relativistic Collisionless Shocks: Fermi Process at Last? Astrophys. J. 682, 5-8 (2008b). doi:10.1086/590248

H.C. Spruit, Theory of Magnetically Powered Jets, in Lecture Notes in Physics, Berlin Springer Verlag, ed. by T. Belloni Lecture Notes in Physics, Berlin Springer Verlag, vol. 794, 2010, p. 233

J.F. Steiner, J.E. McClintock, R. Narayan, Jet Power and Black Hole Spin: Testing an Empirical Relationship and Using it to Predict the Spins of Six Black Holes. Astrophys. J. 762, 104 (2013). doi:10.1088/0004-637X/762/2/104

R.A. Sunyaev, L.G. Titarchuk, Comptonization of X-rays in plasma clouds - Typical radiation spectra. Astron. Astrophys. 86, 121-138 (1980)

H. Tananbaum, H. Gursky, E. Kellogg, R. Giacconi, C. Jones, Observation of a Correlated X-Ray Transition in Cygnus X-1. Astrophys. J. 177, 5 (1972). doi:10.1086/181042

F. Tavecchio, L. Maraschi, R.M. Sambruna, C.M. Urry, The X-Ray Jet of PKS 0637-752: Inverse Compton Radiation from the Cosmic Microwave Background? Astrophys. J. 544, 23-26 (2000). doi:10.1086/317292

A. Tchekhovskoy, R. Narayan, J.C. McKinney, Black Hole Spin and The Radio Loud/Quiet Dichotomy of Active Galactic Nuclei. Astrophys. J. 711, 50-63 (2010). doi:10.1088/0004$637 \mathrm{X} / 711 / 1 / 50$

A. Tchekhovskoy, R. Narayan, J.C. McKinney, Efficient generation of jets from magnetically arrested accretion on a rapidly spinning black hole. Mon. Not. R. Astron. Soc. 418, 79-83 (2011). doi:10.1111/j.1745-3933.2011.01147.x

L. Titarchuk, Generalized Comptonization models and application to the recent high-energy observations. Astrophys. J. 434, 570-586 (1994). doi:10.1086/174760

C.M. Urry, P. Padovani, Unified Schemes for Radio-Loud Active Galactic Nuclei. Publ. Astr. Soc. Pacific 107, 803-845 (1995). doi:10.1086/133630

P. Uttley, T. Wilkinson, P. Cassatella, J. Wilms, K. Pottschmidt, M. Hanke, M. Böck, The causal connection between disc and power-law variability in hard state black hole X-ray binaries. Mon. Not. R. Astron. Soc. 414, 60-64 (2011). doi:10.1111/j.17453933.2011.01056.x

H. van der Laan, A Model for Variable Extragalactic Radio Sources. Nature 211, 1131 (1966). doi:10.1038/2111131a0

A. Veledina, J. Poutanen, I. Vurm, A Synchrotron Self-Compton-Disk Reprocessing Model for Optical/X-Ray Correlation in Black Hole X-Ray Binaries. Astrophys. J. 737, 17 (2011). doi:10.1088/2041-8205/737/1/L17

A. Veledina, J. Poutanen, I. Vurm, Hot accretion flow in black hole binaries: a link 
connecting X-rays to the infrared. Mon. Not. R. Astron. Soc. 430, 3196-3212 (2013). doi:10.1093/mnras/stt124

G.S. Vila, G.E. Romero, Leptonic/hadronic models for electromagnetic emission in microquasars: the case of GX 339-4. Mon. Not. R. Astron. Soc. 403, 1457-1468 (2010). doi:10.1111/j.1365-2966.2010.16208.x

N. Vlahakis, A. Königl, Magnetic Driving of Relativistic Outflows in Active Galactic Nuclei. I. Interpretation of Parsec-Scale Accelerations. Astrophys. J. 605, 656-661 (2004). doi:10.1086/382670

G. Wardziński, A.A. Zdziarski, Thermal synchrotron radiation and its Comptonization in compact X-ray sources. Mon. Not. R. Astron. Soc. 314, 183-198 (2000). doi:10.1046/j.13658711.2000.03297.x

A.E. Wehrle, E. Pian, C.M. Urry, L. Maraschi, I.M. McHardy, A.J. Lawson, G. Ghisellini, R.C. Hartman, et. al., Multiwavelength Observations of a Dramatic High-Energy Flare in the Blazar 3C 279. Astrophys. J. 497, 178 (1998). doi:10.1086/305461

E.S. Weibel, Spontaneously Growing Transverse Waves in a Plasma Due to an Anisotropic Velocity Distribution. Physical Review Letters 2, 83-84 (1959). doi:10.1103/PhysRevLett.2.83

R.A.M.J. Wijers, M.J. Rees, P. Meszaros, Shocked by GRB 970228: the afterglow of a cosmological fireball. Mon. Not. R. Astron. Soc. 288, 51-56 (1997)

Q. Wu, M. Gu, The X-Ray Spectral Evolution in X-Ray Binaries and Its Application to Constrain the Black Hole Mass of Ultraluminous X-Ray Sources. Astrophys. J. 682, 212217 (2008). doi:10.1086/588187

F. Yuan, W. Cui, Radio-X-Ray Correlation and the "Quiescent State" of Black Hole Sources. Astrophys. J. 629, 408-413 (2005). doi:10.1086/431453

F. Yuan, W. Cui, R. Narayan, An Accretion-Jet Model for Black Hole Binaries: Interpreting the Spectral and Timing Features of XTE J1118+480. Astrophys. J. 620, 905-914 (2005). doi:10.1086/427206

F. Yuan, Z. Yu, L.C. Ho, Revisiting the "Fundamental Plane" of Black Hole Activity at Extremely Low Luminosities. Astrophys. J. 703, 1034-1043 (2009). doi:10.1088/0004$637 \mathrm{X} / 703 / 1 / 1034$

F. Yuan, A.A. Zdziarski, Y. Xue, X.-B. Wu, Modeling the Hard States of XTE J1550-564 during Its 2000 Outburst. Astrophys. J. 659, 541-548 (2007). doi:10.1086/512078

A.A. Zdziarski, P. Lubiński, M. Gilfanov, M. Revnivtsev, Correlations between X-ray and radio spectral properties of accreting black holes. Mon. Not. R. Astron. Soc. 342, 355372 (2003). doi:10.1046/j.1365-8711.2003.06556.x

J.F. Zhang, Y.G. Feng, M.C. Lei, Y.Y. Tang, Y.P. Tian, High-energy neutrino emission from low-mass microquasars. Mon. Not. R. Astron. Soc. 407, 2468-2474 (2010). doi:10.1111/j.1365-2966.2010.17072.x

Y.F. Zhang, A.P. Marscher, H.D. Aller, M.F. Aller, H. Terasranta, E. Valtaoja, Radio and $\mathrm{X}$-ray observations of the gamma-ray bright quasar PKS 0528+134. Astrophys. J. 432, 91-102 (1994). doi:10.1086/174551

J. Zrake, A.I. MacFadyen, Numerical Simulations of Driven Relativistic Magnetohydrodynamic Turbulence. Astrophys. J. 744, 32 (2012). doi:10.1088/0004-637X/744/1/32 